NASA/TM-2000-209891, Vol. 47

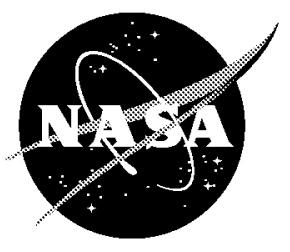

Technical Report Series on the Boreal Ecosystem-Atmosphere Study (BOREAS)

Forrest G. Hall and Jaime Nickeson, Editors

\title{
Volume 47
}

\section{BOREAS RSS-3 Reflectance Measured from a Helicopter-Mounted SE-590}

Charles L. Walthall, U.S.D.A Agricultural Research Service, Beltsville, Maryland Sara Loechel, University of Maryland

National Aeronautics and

Space Administration

Goddard Space Flight Center

Greenbelt, Maryland 20771 
The NASA STI Program Office ... in Profile

Since its founding, NASA has been dedicated to the advancement of aeronautics and space science. The NASA Scientific and Technical Information (STI) Program Office plays a key part in helping NASA maintain this important role.

The NASA STI Program Office is operated by Langley Research Center, the lead center for NASA's scientific and technical information. The NASA STI Program Office provides access to the NASA STI Database, the largest collection of aeronautical and space science STI in the world. The Program Office is also NASA's institutional mechanism for disseminating the results of its research and development activities. These results are published by NASA in the NASA STI Report Series, which includes the following report types:

- TECHNICAL PUBLICATION. Reports of completed research or a major significant phase of research that present the results of NASA programs and include extensive data or theoretical analysis. Includes compilations of significant scientific and technical data and information deemed to be of continuing reference value. NASA's counterpart of peer-reviewed formal professional papers but has less stringent limitations on manuscript length and extent of graphic presentations.

- TECHNICAL MEMORANDUM. Scientific and technical findings that are preliminary or of specialized interest, e.g., quick release reports, working papers, and bibliographies that contain minimal annotation. Does not contain extensive analysis.

- CONTRACTOR REPORT. Scientific and technical findings by NASA-sponsored contractors and grantees.
- CONFERENCE PUBLICATION. Collected papers from scientific and technical conferences, symposia, seminars, or other meetings sponsored or cosponsored by NASA.

- SPECIAL PUBLICATION. Scientific, technical, or historical information from NASA programs, projects, and mission, often concerned with subjects having substantial public interest.

- TECHNICAL TRANSLATION. English-language translations of foreign scientific and technical material pertinent to NASA's mission.

Specialized services that complement the STI Program Office's diverse offerings include creating custom thesauri, building customized databases, organizing and publishing research results ... even providing videos.

For more information about the NASA STI Program Office, see the following:

- Access the NASA STI Program Home Page at http://www.sti.nasa.gov/STI-homepage.html

- E-mail your question via the Internet to help@sti.nasa.gov

- Fax your question to the NASA Access Help Desk at (301) 621-0134

- Telephone the NASA Access Help Desk at (301) 621-0390

- Write to:

NASA Access Help Desk

NASA Center for AeroSpace Information 7121 Standard Drive Hanover, MD 21076-1320 
NASA/TM-2000-209891, Vol. 47

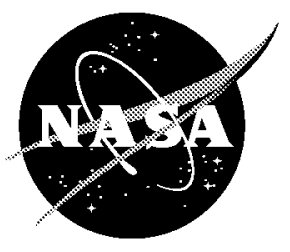

Technical Report Series on the Boreal Ecosystem-Atmosphere Study (BOREAS)

Forrest G. Hall and Jaime Nickeson, Editors

\section{Volume 47}

\section{BOREAS RSS-3 Reflectance Measured from a Helicopter-Mounted SE-590}

Charles L. Walthall, U.S.D.A Agricultural Research Service, Beltsville, Maryland Sara Loechel, University of Maryland

National Aeronautics and

Space Administration

Goddard Space Flight Center

Greenbelt, Maryland 20771 


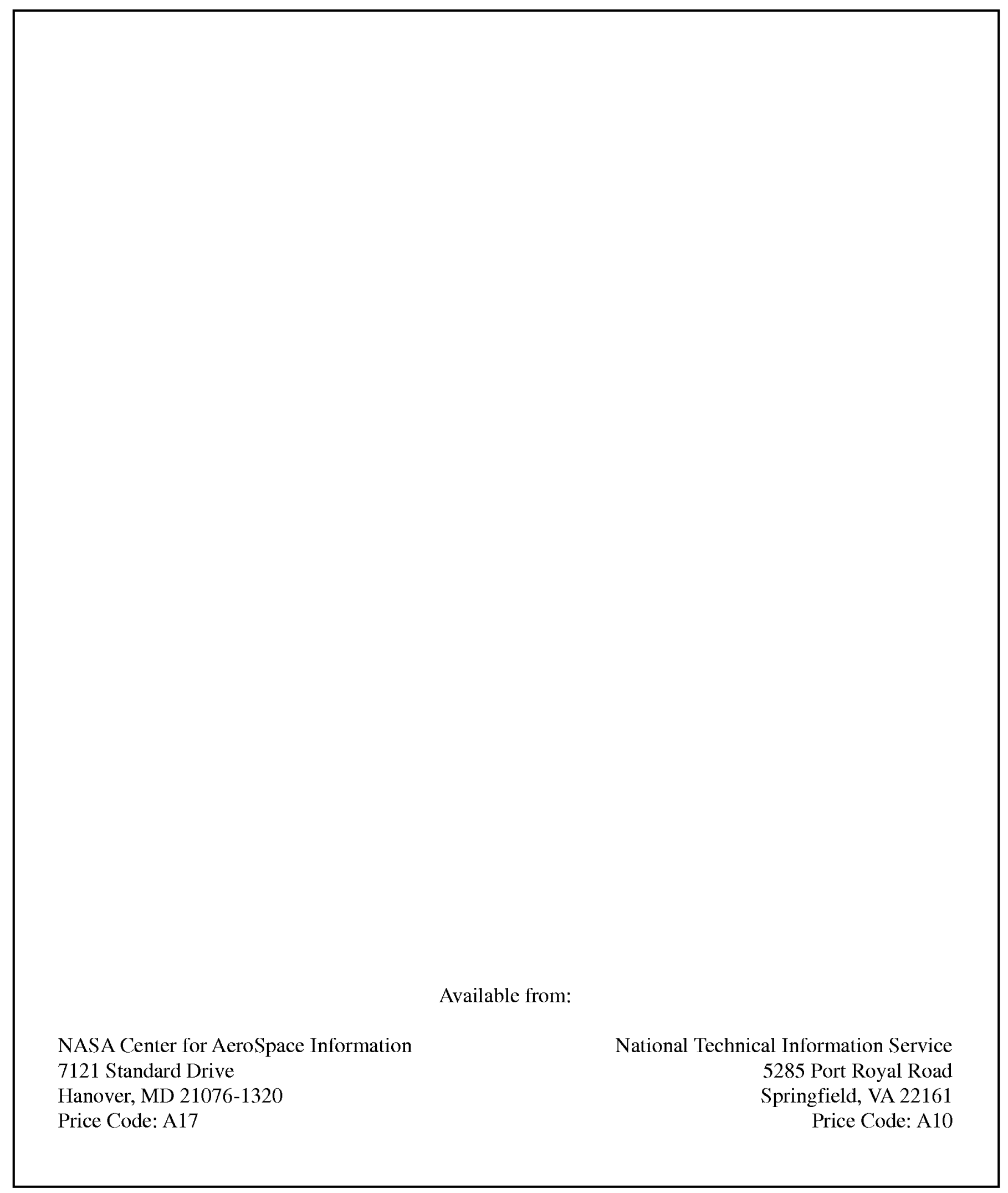




\title{
BOREAS RSS-3 Reflectance Measured from a Helicopter-Mounted SE-590
}

\author{
Charles L. Walthall, Sara Loechel
}

\section{Summary}

The BOREAS RSS-3 team collected multiple remotely sensed data sets from the NASA UH-1 helicopter. This data set includes helicopter-based radiometric measurements of forested sites acquired during BOREAS made with an SE-590 processed to reflectance factors. The data used in this analysis were collected in 1994 during the three BOREAS IFCs at numerous tower and auxiliary sites in both the NSA and the SSA. The 15-degree FOV of the SE-590 yielded a ground resolution of approximately $79 \mathrm{~m}$ at the $300-\mathrm{m}$ nominal altitude. The data are provided in tabular ASCII files.

Note: An extensive helicopter log (in Acrobat format) is available for the 1994 IFC's. Environmental, technical, instrumental, and operational conditions are noted for each observation where applicable. It is strongly recommended that any researcher doing extended work with this data set review this helicopter log.

\section{Table of Contents}

1) Data Set Overview

2) Investigator(s)

3) Theory of Measurements

4) Equipment

5) Data Acquisition Methods

6) Observations

7) Data Description

8) Data Organization

9) Data Manipulations

10) Errors

11) Notes

12) Application of the Data Set

13) Future Modifications and Plans

14) Software

15) Data Access

16) Output Products and Availability

17) References

18) Glossary of Terms

19) List of Acronyms

20) Document Information

\section{Data Set Overview}

\subsection{Data Set Identification}

BOREAS RSS-03 Reflectance Measured from a Helicopter-Mounted SE-590

\subsection{Data Set Introduction}

Radiometer measurements of BOReal Ecosystem-Atmosphere Study (BOREAS) forested tower and auxiliary sites were taken from a helicopter platform at nadir. The data were collected in 1994 during the green-up, peak, and senescent stages of the growing season at numerous tower and auxiliary sites in both the Northern Study Area (NSA) and the Southern Study Area (SSA). The 15-degree field of view (FOV) of the Spectron Engineering Spectroradiometer (SE-590) yielded an 
Instantaneous Field of View (IFOV) of approximately $79 \mathrm{~m}$ from the $300 \mathrm{~m}$ altitude typically flown. The SE-590 has a spectral range of 362.7 to $1122.7 \mathrm{~nm}$, although the "usable" SE-590 range is actually $\sim 400$ to $900 \mathrm{~nm}$. The SE-590 bandwidth is $\sim 15 \mathrm{~nm}$ and the spacing between bands $\sim 3 \mathrm{~nm}$.

The helicopter-measured SE-590 radiances and sunphotometer data were used as input to Version 4.0 of the Second Simulation of the Satellite Signal in the Solar Spectrum (6S) atmospheric correction software to obtain at-surface reflectance factors. The data cover the three Intensive Field Campaign (IFC) periods: 31-May through 10-June (IFC-1), 21-July through 08-August (IFC-2), and 06-September through 16-September (IFC-3).

\subsection{Objective/Purpose}

The objective of the study was to acquire multispectral, bidirectional reflectance data of the study sites for assessments of spectral, spatial, and temporal variability and the impacts of these variabilities on vegetation indices. A helicopter with a pointable stabilized mount was used to carry a spectrometer (visible and near-infrared), a spectroradiometer, an infrared thermometer, and a video camera. An autotracking sunphotometer was also deployed to provide data for calculations of irradiance and for atmospheric correction of the data. The latest available version of the $6 \mathrm{~S}$ atmospheric model was used for the calculations of irradiance and for atmospheric corrections.

\subsection{Summary of Parameters}

Helicopter-based measurements of at-helicopter radiances and standard deviations, at-helicopter and at-surface (atmospherically corrected) reflectances and conditions (surface physical, geometric, and atmospheric) at the time of the observation.

\subsection{Discussion}

These measurements were collected as part of the effort to evaluate models that estimate surface biophysical characteristics from remotely measured optical signatures.

\subsection{Related Data Sets}

BOREAS RSS-01 PARABOLA SSA Surface Reflectance and Transmittance Data

BOREAS RSS-02 Level-1b ASAS Imagery: At-sensor Radiance in BSQ Format

BOREAS RSS-03 Reflectance Measured from a Helicopter-Mounted Barnes MMR

BOREAS RSS-03 Atmospheric Measurements from a Helicopter-Mounted Sunphotometer

BOREAS RSS-03 Video Imagery Acquired from a Helicopter Platform

BOREAS RSS-11 Ground Network of Sun Photometer Measurements

BOREAS RSS-12 Automated Ground Sun Photometer Measurements in the SSA

BOREAS RSS-19 1994 Seasonal Understory Reflectance Data

BOREAS RSS-20 POLDER Measurements of Surface BRDF

\section{Investigator(s)}

\subsection{Investigator(s) Name and Title}

Dr. Charles L. Walthall, Physical Scientist

\subsection{Title of Investigation}

Biophysical Significance of Spectral Vegetation Indices in the Boreal Forest 


\subsection{Contact Information}

\section{Contact 1:}

Dr. Charles L. Walthall

Physical Scientist

USDA Agricultural Research Service

Remote Sensing and Modeling Laboratory

008 Bldg. 007, BARC-West

10300 Baltimore Avenue

Beltsville, MD 20705 USA

(301) 504-6074

(301) 504-5031 (fax)

cwalthal@asrr.arsusda.gov

\section{Contact 2:}

Sara Loechel

Faculty Research Assistant

Department of Geography

University of Maryland

Located at:

USDA Agricultural Research Service

Remote Sensing and Modeling Laboratory

Rm. 008, Bldg. 007, BARC-West

10300 Baltimore Avenue

Beltsville, MD 20705 USA

(301) 504-6823

(301) 504-5031 (fax)

sloechel@asrr.arsusda.gov

\section{Contact 3:}

Jaime Nickeson

Raytheon ITSS

NASA GSFC

Code 923

Greenbelt, MD 20771

(301) 286-3373

(301) 286-0239 (fax)

Jaime.Nickeson@gsfc.nasa.gov

\section{Theory of Measurements}

Radiation striking a vegetative canopy interacts with individual phytoelements (leaves, stems, branches) and the underlying substrate. The interaction depends on light quality, radiative form (direct or diffuse), illumination incidence angle, vegetative component optical properties, and canopy architecture. Radiation is reflected, transmitted, or absorbed.

At-sensor radiances are converted to surface reflectance using the $6 \mathrm{~S}$ atmospheric correction model. Using measurements of optical depth as input, this model accounts for the combined effects of scattering (molecular and aerosol) and absorption by the atmosphere.

The helicopter missions were designed to provide a rapid means of intensive spectral characterization of sites and to provide an intermediate scale of sampling between the surface measurements and the higher altitude aircraft and spacecraft multispectral imaging devices. The SE-590 instrumentation was chosen to provide compatibility with surface-based radiometers and Thematic Mapper (TM) spaceborne sensors. 


\section{Equipment}

\subsection{Sensor/Instrument Description}

The primary instruments for the BOREAS Remote Sensing Science (RSS)-03 deployment are the SE-590, a Barnes Modular Multiband Radiometer (MMR), a color Charge-Coupled Device (CCD)-based video camera, and a sun-tracking photometer. The downward-looking sensor heads, along with a color video camera, are mounted on an operator-controlled pointable mount that gives variability in the view zenith and view azimuth directions independent of the heading of the aircraft.

The SE-590 is a field portable, microprocessor-controlled spectroradiometer developed in the early 1980s. The sensor is a CCD area array detector with a diffraction grating serving as the spectral dispersion component. The complete system consists of a microprocessor-based controller connected by cable to two optical cameras containing the sensors. Lenses are used as collimators for the optical cameras. Although three different optical heads are available for the unit, only two can be used at once with a single controller. The primary unit of interest is the visible/near-infrared (VIS/NIR) optical component, which employs a silicon sensor and is sensitive to radiation in the $400 \mathrm{~nm}$ to $1100 \mathrm{~nm}$ region. The short-wave infrared (SWIR) optical camera uses a lead sulfide detector with a cooling device for temperature stability and is sensitive to radiation in the $1100 \mathrm{~nm}$ to $2500 \mathrm{~nm}$ spectral region. Lenses for 1-degree and 15-degree FOV are available as standard attachments for the VIS/NIR system. A 15 degree FOV lens for the SWIR system was specially fabricated for BOREAS. The serial number of the instrument used was 2071.

For the BOREAS deployment, a temperature-controlled box was built to counter the effects of ambient temperature on radiometric response of the VIS/NIR optical heads. Two VIS/NIR optical heads were housed in the temperature control box for the final configuration, one with a 1-degree FOV lens and one with a 15-degree FOV lens. The SWIR optical head was mounted external to the box with a 15-degree FOV lens. The SWIR head was moved outside the temperature control box to avoid conflicts between its temperature control system and the system of the temperature control box. The configuration of the system was such that two of the three optical heads could be operated at once using a single SE-590 controller. The choice of optical heads (two VIS/NIR or one VIS/NIR and the SWIR) also required a change in software because the data stream from the SWIR optical head is different from that of the VIS/NIR optical heads.

For helicopter use, the SE-590 is operated in a slave mode by a dedicated PC running DOS. The data stream from the SE-590 is communicated via RS-232 cable to the computer, where it is stored on a hard disk. The unit operates on $\mathrm{AC}$ power available from the aircraft via inverters with self-contained rechargeable batteries inside the controller available for backup. Sensor integration time is set automatically via calculations made on a spectral scan prior to each data collection scan.

The data stream from this device includes digital numbers (DNs) for each channel, sensor dwell/integration time, date, time, and maximum signal level in DNs. The PC software adds an operator-specified header and the SE-590 time is replaced with time from the computer's clock as the data are stored.

\subsubsection{Collection Environment}

In general, the helicopter was flown during relatively clear days when possible. Data collection was attempted during conditions of highest possible solar elevation. All observations were attempted from a nadir observation point and usually at $300 \mathrm{~m}$ above ground level (AGL). Exceptions are noted in the helicopter log.

\subsubsection{Source/Platform}

The UH-1 "Huey" series of helicopters has been available as a platform for the system in many field campaigns. The first 10 years of the system development and use were with two UH-1B Huey helicopters, while the aircraft used for BOREAS was a UH-1H model Huey helicopter. Wallops Flight Facility (WFF) changed to the H-model helicopter because of its increased payload capability, the good availability of spare parts, and its widespread use by other organizations. The Bell UH-1H "Iroquois" helicopter, call number N415, was built in 1965 and was acquired by WFF in 1993. Upon acquisition, the aircraft was slightly modified for use as a scientific platform. 
Helicopter N415 operates with standard or low mount, rear-leaning skids. The engine is a Lycoming T53/L13, which provides 1,400 shaft HP with 1,290 transmission HP. The fuel capacity provides 2.0 hours of flying time with a 20 -minute fuel reserve under normal modes of operation. The addition of an auxiliary fuel tank in the port-side door crewman's position provided an additional 15 minutes of flight time during BOREAS given optimum flight conditions.

The instrument platform controllers, power supplies and data loggers are mounted on 54-inch wide, 72-inch-high steel rack mounts fabricated at WFF. Three racks are situated directly in front of the instrument operators. Seats for the instrument operators are located across the front of the transmission and main rotor mast housing. Whenever possible, existing hard points are used for attaching hardware both internally and externally.

The weight of the entire helicopter system with full instrumentation, full fuel, and crew members was 9,500 lbs.

\subsubsection{Source/Platform Mission Objectives}

The helicopter missions were designed to provide a rapid means of intensive spectral characterization of sites and to provide an intermediate scale of sampling between the surface measurements and the higher altitude aircraft and spacecraft multispectral imaging devices. The instruments were chosen to provide compatibility with surface-based radiometers and TM spacecraft sensors.

\subsubsection{Key Variables}

Surface reflectance.

\subsubsection{Principles of Operation}

Computer control of the instruments provides precise, automatic control and ensures proper timing of data collection. The radiometric instruments are configured such that all sensors except the photographic camera can be triggered near-simultaneously with a single computer keyboard keystroke. The command sent from the keyboard is first sent to the SE-590, then to the A/D systems. Raw data from each of the instruments are displayed via graphics and tabular listings on the main computer screen immediately after scanning. The system is configured for multiple sensor data collection. The MMR, SE-590, infrared thermometer, autotracking sunphotometer, and video sensor were the primary payload during BOREAS.

\subsubsection{Sensor/Instrument Measurement Geometry}

The National Aeronautics and Space Administration (NASA) Goddard Space Flight Center (GSFC)/WFF helicopter-based optical remote sensing system was deployed to acquire canopy multispectral data with an SE-590 while hovering approximately 300 meters AGL (Walthall et al., 1996). The 15-degree FOV of the SE-590 yielded a ground resolution of approximately $79 \mathrm{~m}$ at this altitude.

\subsubsection{Manufacturer of Sensor/Instrument}

SE-590:

Spectron Engineering, Inc.

25 Yuma Court

Denver, CO 80223

(303) 733-1060 


\subsection{Calibration}

\subsubsection{Specifications}

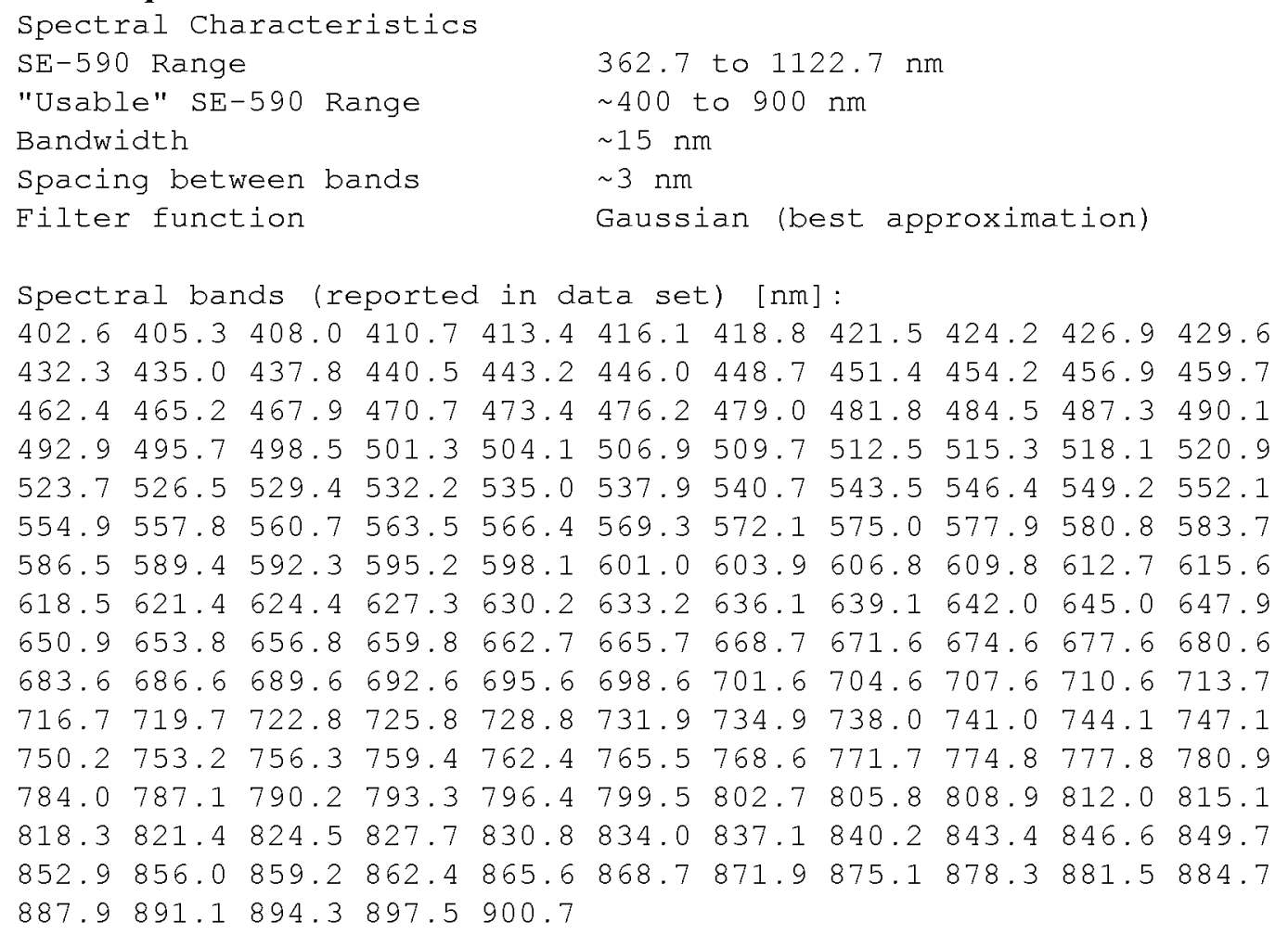

\subsubsection{Tolerance}

None given.

\subsubsection{Frequency of Calibration}

Radiometric calibration and spectral calibration procedures were performed before and after the field season to check for changes in sensor radiometric response. In-field calibration checks were periodically made with a large, portable integrating sphere system. This sphere was used to calibrate the airborne instruments on other aircraft and some of the surface-based radiometric instrumentation.

\subsubsection{Other Calibration Information}

SE-590 coefficients from the second IFC were calculated in the field. Conditions did not change much from the beginning of the first IFC until the end of the last IFC; thus, any of the calibration files are sufficient. The table of calibration coefficients is output from a software module that supplied default wavelengths, instead of the exact wavelengths of this instrument. In practice, they apply to the closest band as given in the SE-590 data set. 
Wavelength Coefficient (offset of zero) and $r^{2}$ from regression

\begin{tabular}{|c|c|c|c|c|c|}
\hline \multicolumn{3}{|c|}{--- - - - - - - - - - - - - - - - - - - - - - - - - - - } & \multicolumn{3}{|c|}{ 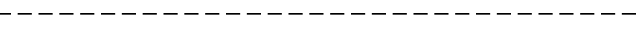 } \\
\hline WAVE & COEF & $r^{2}$ & WAVE & COEF & $r^{2}$ \\
\hline------- & --------- & ---------- & ------ & ------ & --------- \\
\hline 401.5 & 177.877 & 0.944291 & 535.3 & 485.763 & 0.999395 \\
\hline 404.3 & 195.617 & 0.953270 & 538.3 & 486.522 & 0.999378 \\
\hline 407.1 & 212.354 & 0.959176 & 541.2 & 487.035 & 0.999372 \\
\hline 409.9 & 240.441 & 0.969660 & 544.1 & 481.228 & 0.999428 \\
\hline 412.7 & 281.806 & 0.976171 & 547.0 & 471.868 & 0.999408 \\
\hline 415.5 & 329.396 & 0.983505 & 549.9 & 464.951 & 0.999458 \\
\hline 418.3 & 362.033 & 0.985738 & 552.8 & 456.612 & 0.999396 \\
\hline 421.1 & 383.876 & 0.987624 & 555.8 & 454.872 & 0.999417 \\
\hline 423.9 & 406.884 & 0.989234 & 558.7 & 459.347 & 0.999432 \\
\hline 426.7 & 427.885 & 0.990205 & 561.6 & 467.344 & 0.999438 \\
\hline 429.5 & 446.648 & 0.991536 & 564.5 & 478.390 & 0.999478 \\
\hline 432.3 & 454.986 & 0.992297 & 567.5 & 489.023 & 0.999479 \\
\hline 435.1 & 476.587 & 0.994173 & 570.4 & 496.558 & 0.999513 \\
\hline 438.0 & 493.344 & 0.994685 & 573.3 & 505.392 & 0.999555 \\
\hline 440.8 & 511.822 & 0.995202 & 576.3 & 507.749 & 0.999551 \\
\hline 443.6 & 522.264 & 0.995745 & 579.2 & 504.828 & 0.999536 \\
\hline 446.4 & 523.222 & 0.996257 & 582.2 & 498.284 & 0.999541 \\
\hline 449.3 & 519.927 & 0.996496 & 585.1 & 489.910 & 0.999553 \\
\hline 452.1 & 514.144 & 0.997100 & 588.1 & 480.059 & 0.999562 \\
\hline 454.9 & 515.135 & 0.997069 & 591.0 & 470.787 & 0.999550 \\
\hline 457.8 & 507.969 & 0.997500 & 594.0 & 463.137 & 0.999574 \\
\hline 460.6 & 497.376 & 0.997683 & 596.9 & 457.157 & 0.999541 \\
\hline 463.5 & 492.863 & 0.997825 & 599.9 & 454.545 & 0.999552 \\
\hline 466.3 & 492.648 & 0.997897 & 602.9 & 455.709 & 0.999546 \\
\hline 469.2 & 487.060 & 0.998084 & 605.8 & 458.806 & 0.999567 \\
\hline 472.0 & 481.766 & 0.998269 & 608.8 & 463.753 & 0.999581 \\
\hline 474.9 & 478.953 & 0.998335 & 611.8 & 469.958 & 0.999595 \\
\hline 477.7 & 469.324 & 0.998460 & 614.7 & 477.498 & 0.999620 \\
\hline 480.6 & 456.215 & 0.998530 & 617.7 & 482.404 & 0.999643 \\
\hline 483.4 & 447.377 & 0.998650 & 620.7 & 482.992 & 0.999660 \\
\hline 486.3 & 441.005 & 0.998666 & 623.7 & 480.767 & 0.999653 \\
\hline 489.2 & 438.226 & 0.998647 & 626.7 & 477.871 & 0.999653 \\
\hline 492.0 & 441.260 & 0.998873 & 629.6 & 473.047 & 0.999663 \\
\hline 494.9 & 449.451 & 0.998914 & 632.6 & 464.294 & 0.999626 \\
\hline 497.8 & 456.074 & 0.998928 & 635.6 & 456.748 & 0.999657 \\
\hline 500.6 & 459.451 & 0.999010 & 638.6 & 448.964 & 0.999647 \\
\hline 503.5 & 454.181 & 0.999101 & 641.6 & 442.057 & 0.999641 \\
\hline 506.4 & 446.948 & 0.999090 & 644.6 & 436.108 & 0.999634 \\
\hline 509.3 & 440.210 & 0.999098 & 647.6 & 433.334 & 0.999650 \\
\hline 512.2 & 434.007 & 0.999064 & 650.6 & 426.841 & 0.999646 \\
\hline 515.1 & 433.951 & 0.999189 & 653.6 & 424.231 & 0.999654 \\
\hline 518.0 & 434.958 & 0.999175 & 656.6 & 418.953 & 0.999628 \\
\hline 520.8 & 441.795 & 0.999182 & 659.6 & 417.769 & 0.999646 \\
\hline 523.7 & 452.507 & 0.999234 & 662.6 & 419.553 & 0.999671 \\
\hline 526.6 & 461.811 & 0.999292 & 665.7 & 418.793 & 0.999664 \\
\hline 529.5 & 470.346 & 0.999288 & 668.7 & 418.464 & 0.999665 \\
\hline 532.4 & 481.618 & 0.999324 & 671.7 & 418.540 & 0.999691 \\
\hline
\end{tabular}

Page 7 


\begin{tabular}{|c|c|c|}
\hline WAVE & $\mathrm{COEF}$ & $r^{2}$ \\
\hline 674.7 & 419.266 & 0.999675 \\
\hline 677.7 & 414.472 & 0.999683 \\
\hline 680.8 & 408.282 & 0.999666 \\
\hline 683.8 & 402.339 & 0.999697 \\
\hline 686.8 & 394.407 & 0.999663 \\
\hline 689.9 & 383.948 & 0.999669 \\
\hline 692.9 & 377.904 & 0.999662 \\
\hline 695.9 & 371.917 & 0.999645 \\
\hline 699.0 & 366.746 & 0.999641 \\
\hline 702.0 & 362.821 & 0.999658 \\
\hline 705.1 & 364.155 & 0.999659 \\
\hline 708.1 & 361.576 & 0.999668 \\
\hline 711.2 & 364.754 & 0.999664 \\
\hline 714.2 & 370.359 & 0.999676 \\
\hline 717.3 & 372.134 & 0.999673 \\
\hline 720.4 & 376.100 & 0.999686 \\
\hline 723.4 & 381.964 & 0.999712 \\
\hline 726.5 & 383.358 & 0.999717 \\
\hline 729.6 & 385.283 & 0.999733 \\
\hline 732.6 & 387.747 & 0.999745 \\
\hline 735.7 & 389.354 & 0.999746 \\
\hline 738.8 & 385.004 & 0.999735 \\
\hline 741.8 & 379.187 & 0.999740 \\
\hline 744.9 & 371.829 & 0.999722 \\
\hline 748.0 & 363.352 & 0.999721 \\
\hline 751.1 & 354.343 & 0.999710 \\
\hline 754.2 & 347.139 & 0.999680 \\
\hline 757.3 & 340.538 & 0.999703 \\
\hline 760.4 & 336.410 & 0.999688 \\
\hline 763.5 & 329.992 & 0.999674 \\
\hline 766.6 & 325.867 & 0.999690 \\
\hline 769.7 & 319.102 & 0.999647 \\
\hline 772.8 & 311.361 & 0.999645 \\
\hline 775.9 & 305.954 & 0.999653 \\
\hline 779.0 & 299.664 & 0.999634 \\
\hline 782.1 & 296.534 & 0.999644 \\
\hline 785.2 & 292.414 & 0.999615 \\
\hline
\end{tabular}

$\begin{array}{ccc}\text { WAVE } & \text { COEF } & r^{2} \\ ----------------------------------------19 \\ 788.3 & 288.519 & 0.999602 \\ 791.4 & 287.586 & 0.999618 \\ 794.5 & 286.344 & 0.999597 \\ 797.7 & 284.828 & 0.999584 \\ 800.8 & 282.668 & 0.999593 \\ 803.9 & 279.686 & 0.999592 \\ 807.1 & 277.144 & 0.999604 \\ 810.2 & 275.932 & 0.999587 \\ 813.3 & 271.085 & 0.999558 \\ 816.5 & 266.655 & 0.999554 \\ 819.6 & 262.913 & 0.999582 \\ 822.7 & 257.784 & 0.999555 \\ 825.9 & 250.574 & 0.999536 \\ 829.0 & 244.616 & 0.999500 \\ 832.2 & 237.477 & 0.999479 \\ 835.3 & 228.120 & 0.999470 \\ 838.5 & 219.124 & 0.999430 \\ 841.6 & 211.521 & 0.999392 \\ 844.8 & 204.434 & 0.999410 \\ 848.0 & 195.746 & 0.999362 \\ 851.1 & 189.681 & 0.999332 \\ 854.3 & 187.142 & 0.999297 \\ 857.5 & 185.715 & 0.999299 \\ 860.6 & 183.007 & 0.999294 \\ 863.8 & 181.422 & 0.999319 \\ 867.0 & 180.540 & 0.999299 \\ 870.2 & 176.893 & 0.999283 \\ 873.4 & 171.383 & 0.999210 \\ 876.5 & 167.060 & 0.999245 \\ 879.7 & 163.815 & 0.999196 \\ 882.9 & 160.072 & 0.999179 \\ 886.1 & 156.389 & 0.999145 \\ 889.3 & 154.292 & 0.999143 \\ 892.5 & 151.666 & 0.999141 \\ 895.7 & 147.559 & 0.999065 \\ 898.9 & 143.946 & 0.999039 \\ 902.1 & 138.765 & 0.998934 \\ & & \end{array}$

Page 8 


\section{Data Acquisition Methods}

The use of off-the-shelf field instruments aboard airborne platforms is a cost-effective and efficient approach to assembling a data collection system. The instruments are generally rugged enough for the harsh operating environment of a helicopter, provide data comparable to data sets on the surface, and are easy to use and versatile during operation. The system developed jointly at NASA GSFC and WFF uses several widely accepted field-portable radiometric instruments. The system is configured such that instruments from other investigators can be deployed on the helicopter with little or no interference with the primary instrument system. An autotracking sunphotometer system, developed specifically for use on helicopters, is the newest addition to the system.

The NASA GSFC/WFF helicopter-based optical remote sensing system was deployed to acquire canopy multispectral data with an SE-590 while hovering approximately 300 meters AGL (Walthall et al., 1996). The 15-degree FOV of the SE-590 yielded an IFOV at this altitude of approximately $79 \mathrm{~m}$. Observations were made over various tower and auxiliary sites during all three IFCs.

Measurements were collected as conditions permitted during each IFC. In general, the helicopter would hover 1-2 minutes for each observation (consisting of an average number of 20-25 scans).

\section{Observations}

\subsection{Data Notes}

See Section 6.2.

\subsection{Field Notes}

An extensive helicopter log is available for each IFC. Environmental, technical, instrumental, and operational conditions are noted for each observation where applicable.

\section{Data Description}

\subsection{Spatial Characteristics}

\subsubsection{Spatial Coverage}

The helicopter visited all of the NSA and SSA tower and category-1 auxiliary sites. Each site listed below was observed by this instrument at least once during the 1994 campaign at BOREAS. The coordinates provided are based on the North American Datum of 1983 (NAD83).

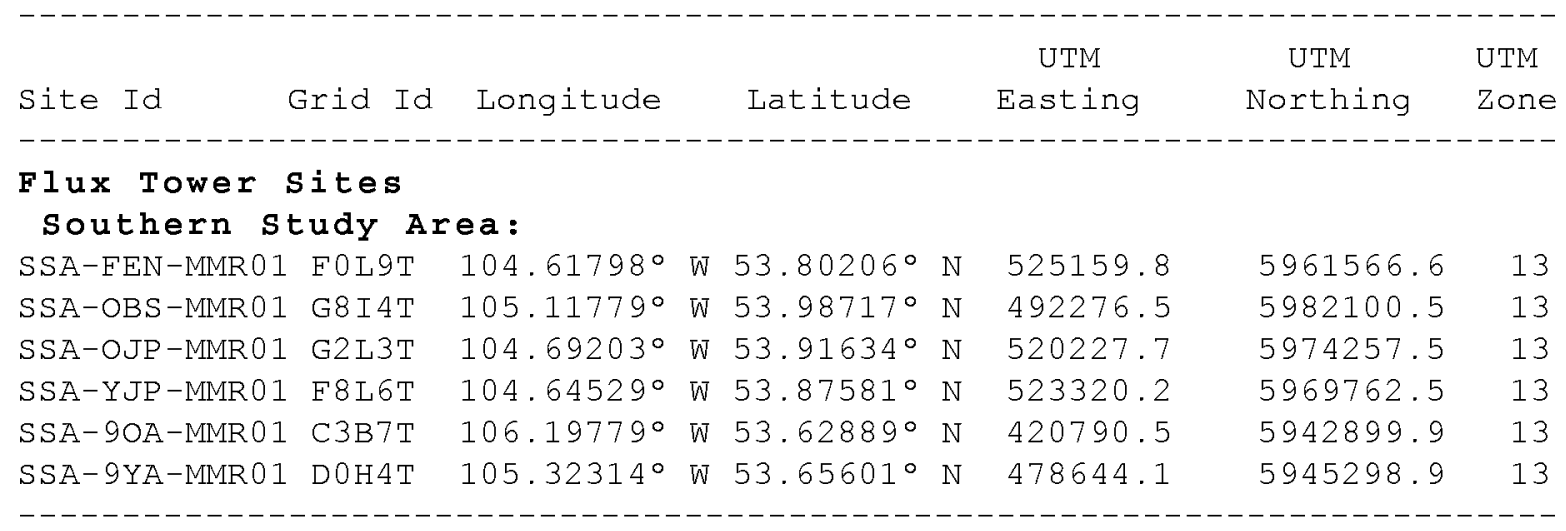




\begin{tabular}{|c|c|c|c|c|c|c|c|c|}
\hline Site Id & Grid Id & Longitude & & Latitude & & $\begin{array}{l}\text { UTM } \\
\text { Easting }\end{array}$ & $\begin{array}{l}\text { UTM } \\
\text { Northing }\end{array}$ & $\begin{array}{l}\text { UTM } \\
\text { Zone }\end{array}$ \\
\hline \multicolumn{9}{|c|}{$\begin{array}{l}\text { Flux Tower Sites } \\
\text { Northern Study Area: }\end{array}$} \\
\hline NSA-OBS-MMRO 1 & $1 \mathrm{~T} 3 \mathrm{R} 8 \mathrm{~T}$ & $98.48139^{\circ}$ & $\bar{W}$ & $55.88007^{\circ}$ & $\mathrm{N}$ & 532444.5 & 6192853.4 & 14 \\
\hline NSA-OJP-MMR 01 & $1 \mathrm{T7Q8T}$ & $98.62396^{\circ}$ & $W$ & $55.92842^{\circ}$ & $\mathrm{N}$ & 523496.2 & 6198176.3 & 14 \\
\hline NSA-YJP-MMR 01 & $1 \mathrm{~T} 8 \mathrm{~S} 9 \mathrm{~T}$ & $98.28706^{\circ}$ & $W$ & $55.89575^{\circ}$ & $\mathrm{N}$ & 544583.9 & 6194706.9 & 14 \\
\hline NSA-BVP-MMR 01 & $1 \mathrm{~T} 4 \mathrm{U} 6 \mathrm{~T}$ & $98.02747^{\circ}$ & $\bar{W}$ & $55.84225^{\circ}$ & $\mathrm{N}$ & 560900.6 & 6188950.7 & 14 \\
\hline NSA-FEN-MMRO 1 & $1 \mathrm{~T} 7 \mathrm{~S} 1 \mathrm{~T}$ & $98.42072^{\circ}$ & $\bar{W}$ & $55.91481^{\circ}$ & $\mathrm{N}$ & 536207.9 & 6196749.6 & 14 \\
\hline \multicolumn{9}{|c|}{$\begin{array}{l}\text { Auxiliary Sites } \\
\text { Southern Study Area: }\end{array}$} \\
\hline SSA-9BS-MMR 01 & 1 D0H6S & $105.29534^{\circ}$ & $W$ & $53.64877^{\circ}$ & $\mathrm{N}$ & 480508.7 & 5944263.4 & 13 \\
\hline SSA-9BS-MMRO 1 & 1 G2I $4 \mathrm{~S}$ & $105.13964^{\circ}$ & $\bar{W}$ & $53.93021^{\circ}$ & $\mathrm{N}$ & 490831.4 & 5975766.3 & 13 \\
\hline SSA-9BS-MMR0I & $1 \mathrm{G} 2 \mathrm{~L} 7 \mathrm{~S}$ & $104.63785^{\circ}$ & $\bar{W}$ & $53.90349^{\circ}$ & $\mathrm{N}$ & 523793.6 & 5972844.3 & 13 \\
\hline SSA-9BS-MMR 01 & 1 G6K8S & $104.75900^{\circ}$ & $W$ & $53.94446^{\circ}$ & $\mathrm{N}$ & 515847.9 & 5977146.9 & 13 \\
\hline$S S A-9 B S-M M R 01$ & 1 G9I4S & $105.11805^{\circ}$ & $W$ & $53.99877^{\circ}$ & $\mathrm{N}$ & 492291.2 & 5983169.1 & 13 \\
\hline SSA-9JP-MMRO 1 & 1 F5I6P & $105.11175^{\circ}$ & $\bar{W}$ & $53.86608^{\circ}$ & $\mathrm{N}$ & 492651.3 & 5968627.1 & 13 \\
\hline SSA-9JP-MMR 01 & 1 F7J0P & $105.05115^{\circ}$ & $\bar{W}$ & $53.88336^{\circ}$ & $\mathrm{N}$ & 496667.0 & 5970323.3 & 13 \\
\hline SSA-9JP-MMR 01 & 1 E7J1P & $105.03226^{\circ}$ & W & $53.88211^{\circ}$ & $\mathrm{N}$ & 497879.4 & 5970405.6 & 13 \\
\hline SSA-9JP-MMRO 1 & $1 \mathrm{G} 1 \mathrm{~K} 9 \mathrm{P}$ & $104.74812^{\circ}$ & $W$ & $53.90880^{\circ}$ & $\mathrm{N}$ & 516546.7 & 5973404.5 & 13 \\
\hline SSA-9JP-MMR 01 & $1 \mathrm{G} 4 \mathrm{~K} 8 \mathrm{P}$ & $104.76401^{\circ}$ & $w$ & $53.91883^{\circ}$ & $\mathrm{N}$ & 515499.1 & 5974516.6 & 13 \\
\hline SSA-9JP-MMR 01 & $1 \mathrm{G} 7 \mathrm{~K} 8 \mathrm{P}$ & $104.77148^{\circ}$ & W & $53.95882^{\circ}$ & $\mathrm{N}$ & 514994.2 & 5978963.8 & 13 \\
\hline SSA-9JP-MMRO 1 & $1 \mathrm{G} 8 \mathrm{~L} 6 \mathrm{P}$ & $104.63755^{\circ}$ & 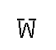 & $53.96558^{\circ}$ & $\mathrm{N}$ & 523778.0 & 5979752.7 & 13 \\
\hline SSA-9JP-MMR 01 & 1 G9L0P & $104.73779^{\circ}$ & $w$ & $53.97576^{\circ}$ & $\mathrm{N}$ & 517197.7 & 5980856.0 & 13 \\
\hline SSA-9JP-MMR 01 & 1 I 2 I $8 \mathrm{P}$ & $105.05107^{\circ}$ & $\mathrm{W}$ & $54.11181^{\circ}$ & $\mathrm{N}$ & 496661.4 & 5995963.1 & 13 \\
\hline SSA-ASP-MMRO 1 & 1 B9B7A & $106.18693^{\circ}$ & $W$ & $53.59098^{\circ}$ & $\mathrm{N}$ & 421469.8 & 5938447.2 & 13 \\
\hline SSA-ASP-MMR 01 & $1 \mathrm{D} 6 \mathrm{H} 4 \mathrm{~A}$ & $105.31546^{\circ}$ & $W$ & $53.70828^{\circ}$ & $\mathrm{N}$ & 479177.5 & 5951112.1 & 13 \\
\hline SSA-ASP-MMR 01 & $1 \mathrm{D} 6 \mathrm{I} 9 \mathrm{~A}$ & $104.63880^{\circ}$ & $W$ & $53.66879^{\circ}$ & $\mathrm{N}$ & 523864.0 & 5946733.2 & 13 \\
\hline SSA-ASP-MMR 01 & $1 \mathrm{D} 9 \mathrm{G} 4 \mathrm{~A}$ & $105.46929^{\circ}$ & W & $53.74019^{\circ}$ & $\mathrm{N}$ & 469047.1 & 5954718.4 & 13 \\
\hline SSA-MIX-MMROI & $1 \mathrm{D} 9 \mathrm{I} 1 \mathrm{M}$ & $105.20643^{\circ}$ & $w$ & $53.72540^{\circ}$ & $\mathrm{N}$ & 486379.7 & 5952989.7 & 13 \\
\hline SSA-MIX-MMR 01 & 1 F1NOM & $104.53300^{\circ}$ & $w$ & $53.80594^{\circ}$ & $\mathrm{N}$ & 530753.7 & 5962031.8 & 13 \\
\hline SSA-MIX-MMRO 1 & 1 G4I3M & $105.14246^{\circ}$ & W & $53.93750^{\circ}$ & $\mathrm{N}$ & 490677.3 & 5976354.9 & 13 \\
\hline
\end{tabular}

Page 10 


\begin{tabular}{|c|c|c|c|c|c|c|c|c|}
\hline Site Id & Grid Id & Longitude & & Latitude & & $\begin{array}{l}\text { UTM } \\
\text { Easting }\end{array}$ & $\begin{array}{l}\text { UTM } \\
\text { Northing }\end{array}$ & $\begin{array}{l}\text { UTM } \\
\text { Zone }\end{array}$ \\
\hline \multicolumn{9}{|c|}{$\begin{array}{l}\text { Auxiliary Sites } \\
\text { Northern study Area: }\end{array}$} \\
\hline NSA-9BS-MMRO 1 & S S 8 WOS & $97.84024^{\circ}$ & $W$ & $55.76824^{\circ}$ & $\mathrm{N}$ & 572761.9 & 6180894.9 & 14 \\
\hline NSA-9BS-MMR0 1 & $1 \mathrm{TOP} 7 \mathrm{~S}$ & $98.82345^{\circ}$ & $W$ & $55.88371^{\circ}$ & $\mathrm{N}$ & 511043.9 & 6193151.1 & 14 \\
\hline NSA-9BS-MMR 01 & $1 \mathrm{~T} 0 \mathrm{P} 8 \mathrm{~S}$ & $98.80225^{\circ}$ & $W$ & $55.88351^{\circ}$ & $\mathrm{N}$ & 512370.1 & 6193132.0 & 14 \\
\hline NSA-9BS-MMR0 1 & 1 T0W1S & $97.80937^{\circ}$ & $w$ & $55.78239^{\circ}$ & $\mathrm{N}$ & 574671.7 & 6182502.0 & 14 \\
\hline NSA-9BS-MMR0 1 & 1 T3U9S & $97.98339^{\circ}$ & $w$ & $55.83083^{\circ}$ & $\mathrm{N}$ & 563679.1 & 6187719.2 & 14 \\
\hline NSA-9BS-MMR 01 & $1 \mathrm{~T} 4 \mathrm{U} 8 \mathrm{~S}$ & $97.99325^{\circ}$ & $w$ & $55.83913^{\circ}$ & $\mathrm{N}$ & 563048.2 & 6188633.4 & 14 \\
\hline NSA-9BS-MMR0 1 & 1 T4U9S & $97.98364^{\circ}$ & $w$ & $55.83455^{\circ}$ & $\mathrm{N}$ & 563657.5 & 6188132.8 & 14 \\
\hline NSA-9BS-MMR 01 & $1 \mathrm{~T} 527 \mathrm{~S}$ & $98.64022^{\circ}$ & $w$ & $55.91610^{\circ}$ & $\mathrm{N}$ & 522487.2 & 6196800.5 & 14 \\
\hline NSA-9BS-MMR 01 & $1 \mathrm{~T} 6 \mathrm{R} 5 \mathrm{~S}$ & $98.51865^{\circ}$ & $w$ & $55.90802^{\circ}$ & $\mathrm{N}$ & 530092.0 & 6195947.0 & 14 \\
\hline NSA-9BS-MMR 01 & $1 \mathrm{~T} 6 \mathrm{~T} 6 \mathrm{~S}$ & $98.18658^{\circ}$ & $w$ & $55.87968^{\circ}$ & $\mathrm{N}$ & 550887.9 & 6192987.9 & 14 \\
\hline NSA-9BS-MMR 01 & 1 T7R9S & $98.44877^{\circ}$ & $w$ & $55.91506^{\circ}$ & $\mathrm{N}$ & 534454.5 & 6196763.6 & 14 \\
\hline NSA-9BS-MMR 01 & 1 T7T3S & $98.22621^{\circ}$ & $w$ & $55.89358^{\circ}$ & $\mathrm{N}$ & 548391.8 & 6194505.6 & 14 \\
\hline NSA-9BS-MMR0 1 & $1 \mathrm{~T} 8 \mathrm{~S} 4 \mathrm{~S}$ & $98.37111^{\circ}$ & $W$ & $55.91689^{\circ}$ & $\mathrm{N}$ & 539306.4 & 6197008.6 & 14 \\
\hline NSA-9BS-MMR0 1 & 1 U5W5S & $97.70986^{\circ}$ & $\bar{W}$ & $55.90610^{\circ}$ & $\mathrm{N}$ & 580655.5 & 6196380.8 & 14 \\
\hline NSA-9BS-MMR 01 & 1 U6W5S & $97.70281^{\circ}$ & $w$ & $55.91021^{\circ}$ & $\mathrm{N}$ & 581087.8 & 6196846.5 & 14 \\
\hline NSA $-9 \mathrm{JP}-\mathrm{MMR} 01$ & $19909 \mathrm{P}$ & $99.03952^{\circ}$ & $W$ & $55.88173^{\circ}$ & $\mathrm{N}$ & 497527.8 & 6192917.5 & 14 \\
\hline NSA-9JP-MMRO 1 & 1 Q3V3P & $98.02473^{\circ}$ & $w$ & $55.55712^{\circ}$ & $\mathrm{N}$ & 561517.9 & 6157222.2 & 4 \\
\hline NSA-9JP-MMR 01 & 1 T7S9P & $98.30037^{\circ}$ & $w$ & $55.89486^{\circ}$ & $\mathrm{N}$ & 543752.4 & 6194599.1 & 4 \\
\hline NSA $-9 \mathrm{JP}-\mathrm{MMR} 01$ & $1 \mathrm{~T} 8 \mathrm{Q9P}$ & $98.61050^{\circ}$ & $w$ & $55.93219^{\circ}$ & $\mathrm{N}$ & 524334.5 & 6198601.4 & 14 \\
\hline NSA-9JP-MMRO 1 & $1 \mathrm{~T} 8 \mathrm{~S} 9 \mathrm{P}$ & $98.28385^{\circ}$ & $\bar{W}$ & $55.90456^{\circ}$ & $\mathrm{N}$ & 544774.3 & 6195688.9 & 4 \\
\hline NSA-9JP-MMR 01 & $1 \mathrm{~T} 8 \mathrm{~T} 1 \mathrm{P}$ & $98.26269^{\circ}$ & $w$ & $55.90539^{\circ}$ & $\mathrm{N}$ & 546096.3 & 6195795.3 & 14 \\
\hline NSA-9JP-MMR 01 & $1 \mathrm{~T} 9 \mathrm{QPP}$ & $98.59568^{\circ}$ & $w$ & $55.93737^{\circ}$ & $\mathrm{N}$ & 525257.1 & 6199183.2 & 4 \\
\hline NSA-9OA-MMRO 1 & $1 \mathrm{~T} 2 \mathrm{Q} 6 \mathrm{~A}$ & $98.67479^{\circ}$ & $\bar{W}$ & $55.88691^{\circ}$ & $\mathrm{N}$ & 520342.0 & 6193540.7 & 14 \\
\hline NSA-ASP-MMR 01 & 1 P7VIA & $07478^{\circ}$ & $w$ & $55.50253^{\circ}$ & $\mathrm{N}$ & 558442.1 & 6151103.7 & 14 \\
\hline NSA-ASP-MMR 01 & 1 Q3V2A & $02635^{\circ}$ & $w$ & $55.56227^{\circ}$ & $\mathrm{N}$ & 561407.9 & 6157793.5 & 14 \\
\hline NSA-ASP-MMR 01 & 1 R8V8A & $97.89260^{\circ}$ & $\bar{W}$ & $55.67779^{\circ}$ & $\mathrm{N}$ & 569638.4 & 6170774.8 & 14 \\
\hline NSA-ASP-MMR 01 & 1 S9P3A & $87621^{\circ}$ & $w$ & $55.88576^{\circ}$ & $\mathrm{N}$ & 507743.3 & 6193371.6 & 14 \\
\hline NSA-ASP-MMR 01 & $1 \mathrm{~T} 4 \mathrm{U} 5 \mathrm{~A}$ & $98.04329^{\circ}$ & $w$ & $55.84757^{\circ}$ & $\mathrm{N}$ & 559901.6 & 6189528.2 & 14 \\
\hline NSA-ASP-MMR 01 & $1 \mathrm{~T} 8 \mathrm{~S} 4 \mathrm{~A}$ & $98.37041^{\circ}$ & $\bar{w}$ & $55.91856^{\circ}$ & $\mathrm{N}$ & 539348.3 & 6197194.6 & 14 \\
\hline NSA-ASP-MMR 01 & $1 \mathrm{~V} 5 \times 7 \mathrm{~A}$ & $97.48565^{\circ}$ & $\bar{W}$ & $55.97396^{\circ}$ & $\mathrm{N}$ & 594506.1 & 6204216.6 & 14 \\
\hline NSA-ASP-MMR 01 & 1 WOY $5 A$ & $97.33550^{\circ}$ & $w$ & $56.00339^{\circ}$ & $\mathrm{N}$ & 603796.6 & 6207706.6 & 14 \\
\hline NSA-MIX-MMR 01 & $1 \mathrm{Q} 1 \mathrm{~V} 2 \mathrm{M}$ & $98.03769^{\circ}$ & $\bar{W}$ & $55.54568^{\circ}$ & $\mathrm{N}$ & 560718.3 & 6155937.3 & 14 \\
\hline NSA-MIX-MMR 01 & 1 TOP5M & $98.85662^{\circ}$ & $w$ & $55.88911^{\circ}$ & $\mathrm{N}$ & 508967.7 & 6193747.3 & 14 \\
\hline
\end{tabular}

\subsubsection{Spatial Coverage Map Not available.}

\subsubsection{Spatial Resolution}

The 15-degree FOV of the SE-590 yielded a ground resolution of $79 \mathrm{~m}$ from the $300 \mathrm{~m}$ altitude.

\subsubsection{Projection \\ Not applicable.}

\subsubsection{Grid Description Not applicable.}




\subsection{Temporal Characteristics}

\subsubsection{Temporal Coverage}

Observations were made during all three BOREAS 1994 IFCs, which occurred during the following periods:

- IFC-1 24-May - 16-June

- $\quad$ IFC-2 19-July - 10-August

- IFC-3 30-August - 19-September

Measurements were made as conditions permitted during each IFC.

\subsubsection{Temporal Coverage Map}

Observations were made at several sites on the following dates:

$\begin{array}{cc}\text { Date } & \text { Study Area } \\ \text { 31-May-1994 } & \text { SSA } \\ 01-J u n-1994 & \text { SSA } \\ 04-J u n-1994 & \text { SSA } \\ 06-J u n-1994 & \text { SSA } \\ 07-J u n-1994 & \text { SSA } \\ 08-J u n-1994 & \text { NSA } \\ 10-J u n-1994 & \text { NSA } \\ 21-J u 1-1994 & \text { NSA } \\ 22-J u 1-1994 & \text { SSA } \\ 23-J u 1-1994 & \text { SSA } \\ 24-J u 1-1994 & \text { SSA } \\ 25-J u 1-1994 & \text { SSA } \\ 28-J u 1-1994 & \text { SSA } \\ 04-A u g-1994 & \text { NSA } \\ 08-A u g-1994 & \text { NSA } \\ 06-S e p-1994 & \text { NSA } \\ 08-S e p-1994 & \text { NSA } \\ 09-S e p-1994 & \text { NSA } \\ 13-S e p-1994 & \text { NSA } \\ 15-S e p-1994 & \text { SSA } \\ 16-S e p-1994 & \text { SSA } \\ & \end{array}$

\subsubsection{Temporal Resolution}

Measurements were collected as conditions permitted during each IFC. In general, the helicopter would hover 1-2 minutes for each observation (consisting of an average number of 20-25 scans). Each site was visited as often as possible during each IFC, with priority given to tower flux sites and category 1 auxiliary sites. Helicopter flight time was limited to approximately 2 hours by fuel constraints. As many sites as possible were visited during each flight.

\subsection{Data Characteristics}




\subsubsection{Parameter/Variable}

The parameters contained in the data files on the CD-ROM are:

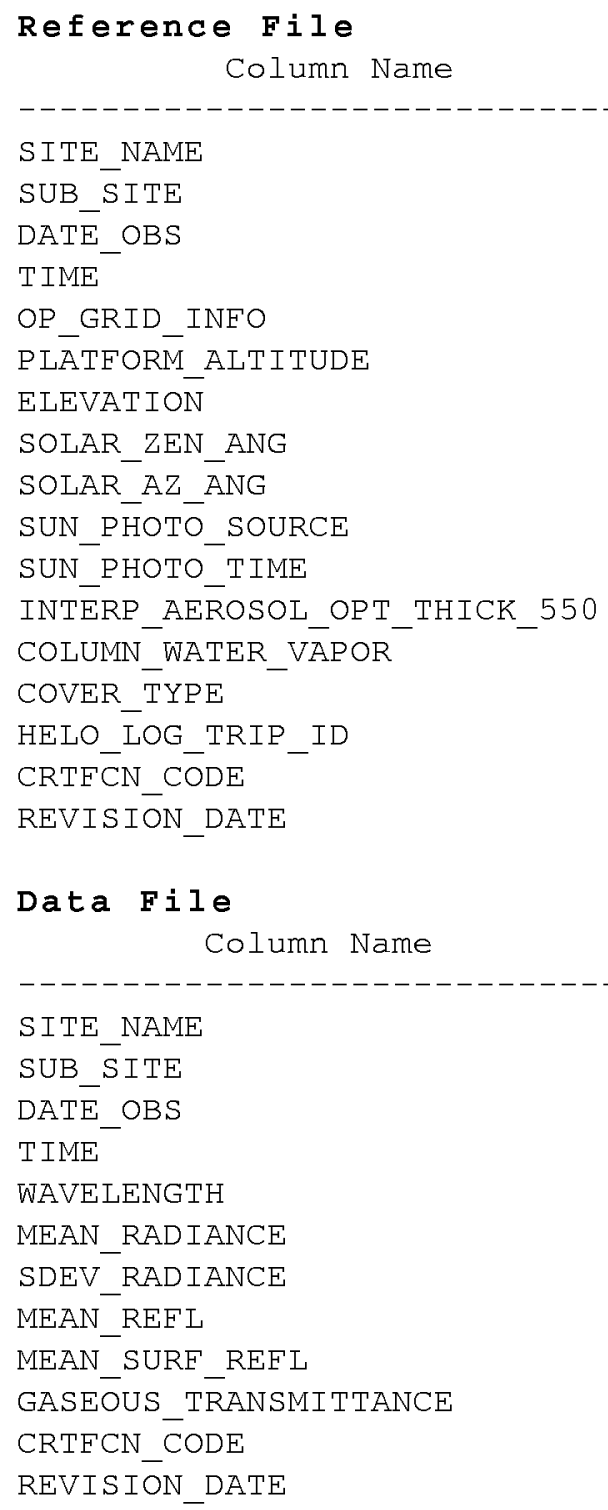




\subsubsection{Variable Description/Definition \\ The descriptions of the parameters contained in the data files on the CD-ROM are:}

Reference File

Column Name

SITE_NAME

SUB_SITE

DATE_OBS

TIME

OP_GRID_INFO

PLATFORM_ALTITUDE

ELEVATION

SOLAR_ZEN_ANG

SOLAR_AZ_ANG

SUN_PHOTO_SOURCE

SUN_PHOTO_TIME

INTERP_AEROSOL_OPT_THICK_550

COLUMN_WATER_VAPOR

COVER_TYPE

HELO_LOG_TRIP_ID

CRTFCN_CODE
Description

The identifier assigned to the site by BOREAS, in the format SSS-TTT-CCCCC, where SSS identifies the portion of the study area: NSA, SSA, REG, TRN, and TTT identifies the cover type for the site, 999 if unknown, and CCCCC is the identifier for site, exactly what it means will vary with site type.

The identifier assigned to the sub-site by BOREAS, in the format GGGGG-IIIII, where GGGGG is the group associated with the sub-site instrument e.g. HYD06 or STAFF, and IIIII is the identifier for sub-site, often this will refer to an instrument.

The date on which the data were collected.

The Greenwich Mean Time (GMT) when the data were collected.

The site identifier used by this RSS team during the execution of field operations. This is used to further link data to site information contained elsewhere.

The nominal altitude of the data collection platform above the target.

The elevation of the site above mean sea level. The angle from the surface normal (straight up) to the sun during data collection.

Direction referred to as a circular scale of degrees read clockwise describing the position of the sun where 0 =north, 90 =east, 180 =south, and $270=$ west.

Indicator of the source of the sun photometer measurements used in atmospheric correction, where helo=helicopter sunphotometer, NSA-YJP = NSA-YJP tower site, Thom = Thompson airport (NSA), SSA-YJP = SSA-YJP tower site.

The time of the sun photometer measurement used in atmospheric correction.

The aerosol optical depth interpolated to 550 nanometers, used in the atmospheric correction. The amount of precipitable water within a vertical column of air with a cross-section of 1 centimeter squared and a fixed depth (usually from the ground to the top of the atmosphere). The dominant species, vegetation or type of land cover that exists at the location. Identifier, given as Mon-Day-(A/B/C), whose letter identifies the particular flight on a given day, used in the helo log file. The BOREAS certification level of the data. 
Examples are CPI (Checked by PI), CGR (Certified by Group), PRE (Preliminary), and CPI-??? (CPI but questionable).

REVISION_DATE

The most recent date when the information in the referenced data base table record was revised.

Data File

Column Name

Description

SITE_NAME

The identifier assigned to the site by BOREAS, in the format SSS-TTT-CCCCC, where SSS identifies the portion of the study area: NSA, SSA, REG, TRN, and TTT identifies the cover type for the site, 999 if unknown, and CCCCC is the identifier for site, exactly what it means will vary with site type.

SUB_SITE

The identifier assigned to the sub-site by

BOREAS, in the format GGGGG-IIIII, where GGGGG is the group associated with the sub-site

instrument e.g. HYD06 or STAFF, and IIIII is the identifier for sub-site, often this will refer to an instrument.

DATE_OBS

The date on which the data were collected.

TIME

The Greenwich Mean Time (GMT) when the data were collected.

WAVELENGTH

Spectral wavelength at which measurement was acquired.

MEAN RADIANCE

The mean at-sensor radiance.

SDEV RADIANCE

The standard deviation at-sensor radiance.

MEAN REFI

MEAN SURF REFL

The mean reflectance factor.

The mean surface reflectance factor

(atmospherically corrected).

GASEOUS TRANSMITTANCE

One minus the absorptance and reflectance of the atmosphere (two-way) due to global gases

(primarily water vapor and ozone).

CRTFCN_CODE

The BOREAS certification level of the data. Examples are CPI (Checked by PI), CGR (Certified by Group), PRE (Preliminary), and CPI-??? (CPI but questionable).

REVISION_DATE

The most recent date when the information in the referenced data base table record was revised.

The OP_GRID_INFO in the SE590 reference data are tightly linked to the Helicopter log documentation; much information relevant to the location of the observation and the conditions at the time are available in the log. 


\subsubsection{Unit of Measurement}

The measurement units for the parameters contained in the data files on the CD-ROM are:

Reference File

Column Name

SITE NAME

SUB SITE

DATE OBS

TIME

OP_GRID_INFO

PLATFORM ALTITUDE

ELEVATION

SOLAR_ZEN_ANG

SOLAR AZ ANG

SUN_PHOTO_SOURCE

SUN_PHOTO_TIME

INTERP_AEROSOL_OPT_THICK_550

COLUMN WATER VAAPOR

COVER TYPE

HELO_LOG TRIP_ID

CRTECN_CODE

REVISION_DATE
Units

[none]

[none]

[DD-MON-YY]

[HHMMSS GMT]

[none ]

[meters ]

[meters]

[degrees]

[degrees ]

[none]

[HHMMSS GMT]

[unitless]

[millimeters]

[none ]

[none]

[none ]

[DD-MON-YY]

Data File

Column Name

SITE NAME

SUB_SITE

DATE_OBS

TIME

WAVELENGTH

MEAN RADIANCE

SDEV RADIANCE

MEAN_REEI

MEAN_SURF REFI

GASEOUS TRANSMITTANCE

CRTFCN_CODE

REVISION_DATE
Units

[none ]

[none ]

[DD-MON-YY]

[HHMMSS GMT]

[micrometers ]

[Watts] [meter^-2] [steradian^-1] [micrometer^-1]

[Watts ] [meter ${ }^{\wedge}-2$ ] [steradian^ -1 ] [micrometer ${ }^{\wedge}-1$ ]

[percent]

[percent]

[unitless]

[none ]

$[\mathrm{DD}-\mathrm{MON}-\mathrm{YY}]$

\subsubsection{Data Source}

The sources of the parameter values contained in the data files on the CD-ROM are:

Reference File

Column Name

SITE_NAME

SUB_SITE

DATE OBS

TIME

OP_GRID_INFO

PLATFORM_ALTITUDE

ELEVATION

SOLAR_ZEN_ANG
Data Source

[Assigned by BORIS Staff]

[Assigned by BORIS Staff]

[Controller]

[Controller]

[RSSO3 team]

[NASA Helicopter]

[Experiment Plan]

[Calculated by software] 


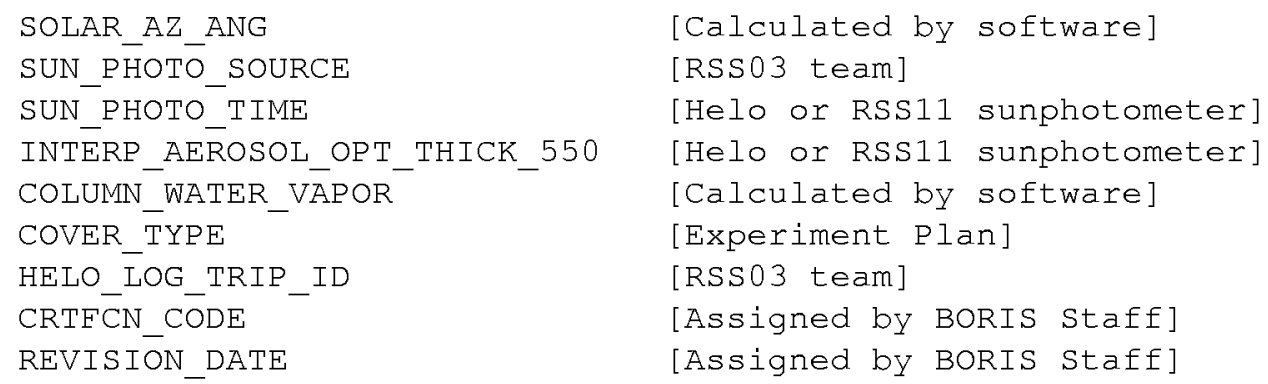

Data File

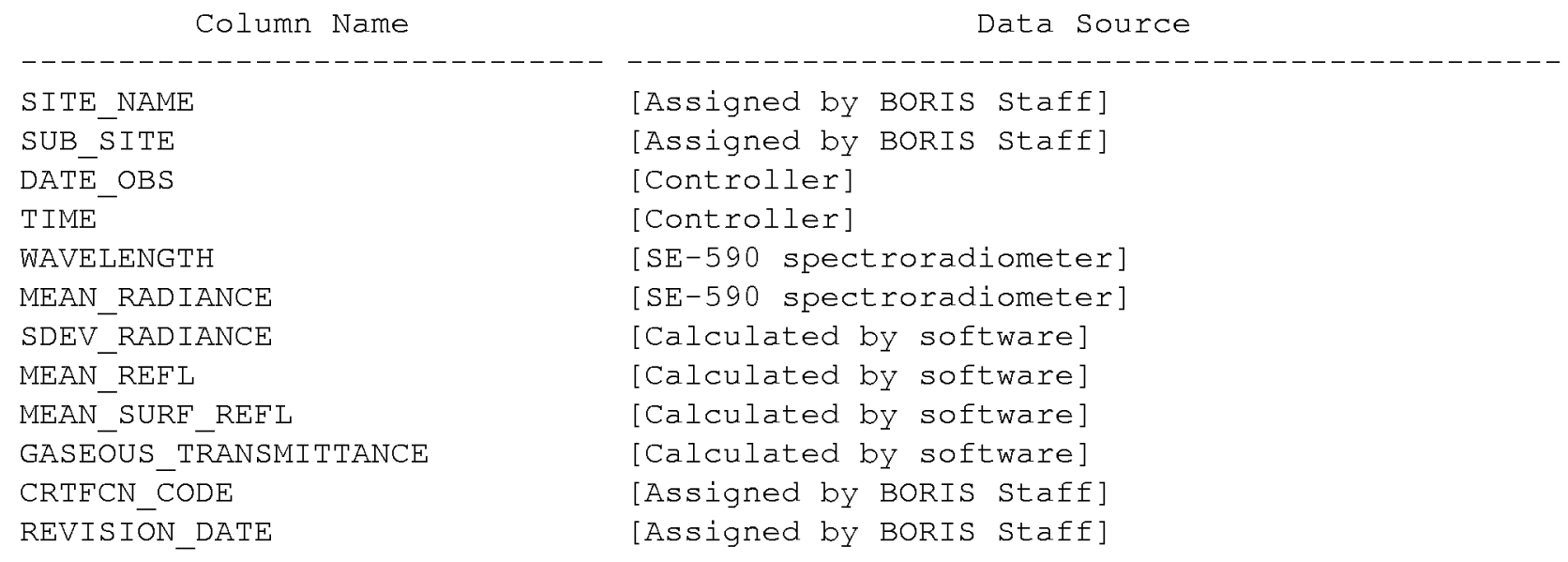

7.3.5 Data Range

The following table gives information about the parameter values found in the data files on the CD-ROM.

Reference File

\begin{tabular}{|c|c|c|c|c|c|c|}
\hline Column Name & $\begin{array}{l}\text { Minimum } \\
\text { Data } \\
\text { Value }\end{array}$ & $\begin{array}{l}\text { Maximum } \\
\text { Data } \\
\text { Value }\end{array}$ & $\begin{array}{l}\text { Missng } \\
\text { Data } \\
\text { Value }\end{array}$ & $\begin{array}{l}\text { Unrel } \\
\text { Data } \\
\text { Value }\end{array}$ & $\begin{array}{l}\text { Below } \\
\text { Detect } \\
\text { Limit }\end{array}$ & $\begin{array}{l}\text { Data } \\
\text { Not } \\
\text { Cllctd }\end{array}$ \\
\hline \multicolumn{7}{|c|}{-- - - - - - - - - - - - - - - - - - - - - - - - - - - - - - - - - - - - - - - - - - - - - - - - - - - - - - - - - - - - - - - - - - } \\
\hline SITE_NAME & NSA-9BS-9TETR & $S S A-Y J P-F L X T R$ & None & None & None & None \\
\hline SUB_SITE & RSS $03-S E 501$ & RSS03-SE501 & None & None & None & None \\
\hline DATE_OBS & $31-M A Y-94$ & $16-\mathrm{SEP}-94$ & None & None & None & None \\
\hline TIME & 143212 & 225100 & None & None & None & None \\
\hline OP_GRID_INFO & $\mathrm{N} / \mathrm{A}$ & $N / A$ & None & None & None & None \\
\hline PLATEORM_ALTITUDE & 304.8 & 304.8 & None & None & None & None \\
\hline ELEVATION & 136 & 650.44 & None & None & None & None \\
\hline SOLAR_ZEN_ANG & 32.827 & 63.215 & None & None & None & None \\
\hline SOLAR_AZ_ANG & 96.059 & 253.65 & None & None & None & None \\
\hline SUN_PHOTO_SOURCE & Helo & Thom & None & None & None & None \\
\hline SUN_PHOTO_TIME & 143300 & 223200 & None & None & None & None \\
\hline $\begin{array}{l}\text { INTERP_AEROSOL_OPT_ } \\
\text { THICK_550 }\end{array}$ & .0315 & .7464 & None & None & None & None \\
\hline COLUMN_WATER_VAPOR & 6.35 & 27.01 & None & None & None & None \\
\hline COVER_TYPE & $N / A$ & $\mathrm{~N} / \mathrm{A}$ & None & None & None & None \\
\hline HELO_LOG_TRIP_ID & $531 \mathrm{~A}$ & $916 \mathrm{~B}$ & None & None & None & None \\
\hline CRTFCN_CODE & $\mathrm{CPI}$ & $\mathrm{CPI}$ & None & None & None & None \\
\hline REVISION_DATE & $13-J U N-98$ & $13-J U N-98$ & None & None & None & None \\
\hline
\end{tabular}




\section{Data File}

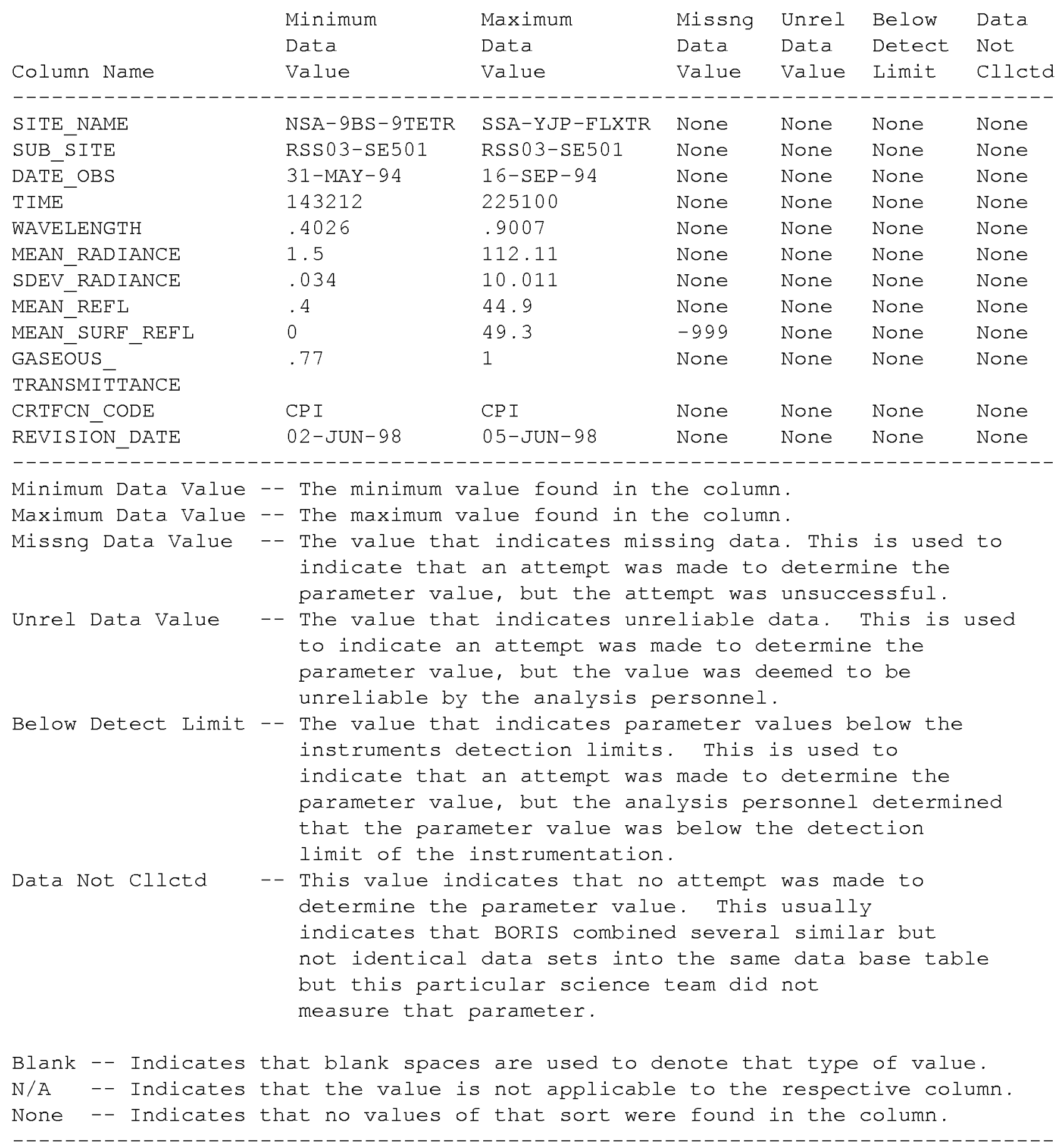

\section{Page 18}




\subsection{Sample Data Record}

The following is a sample of the first few records from the data table on the CD-ROM:

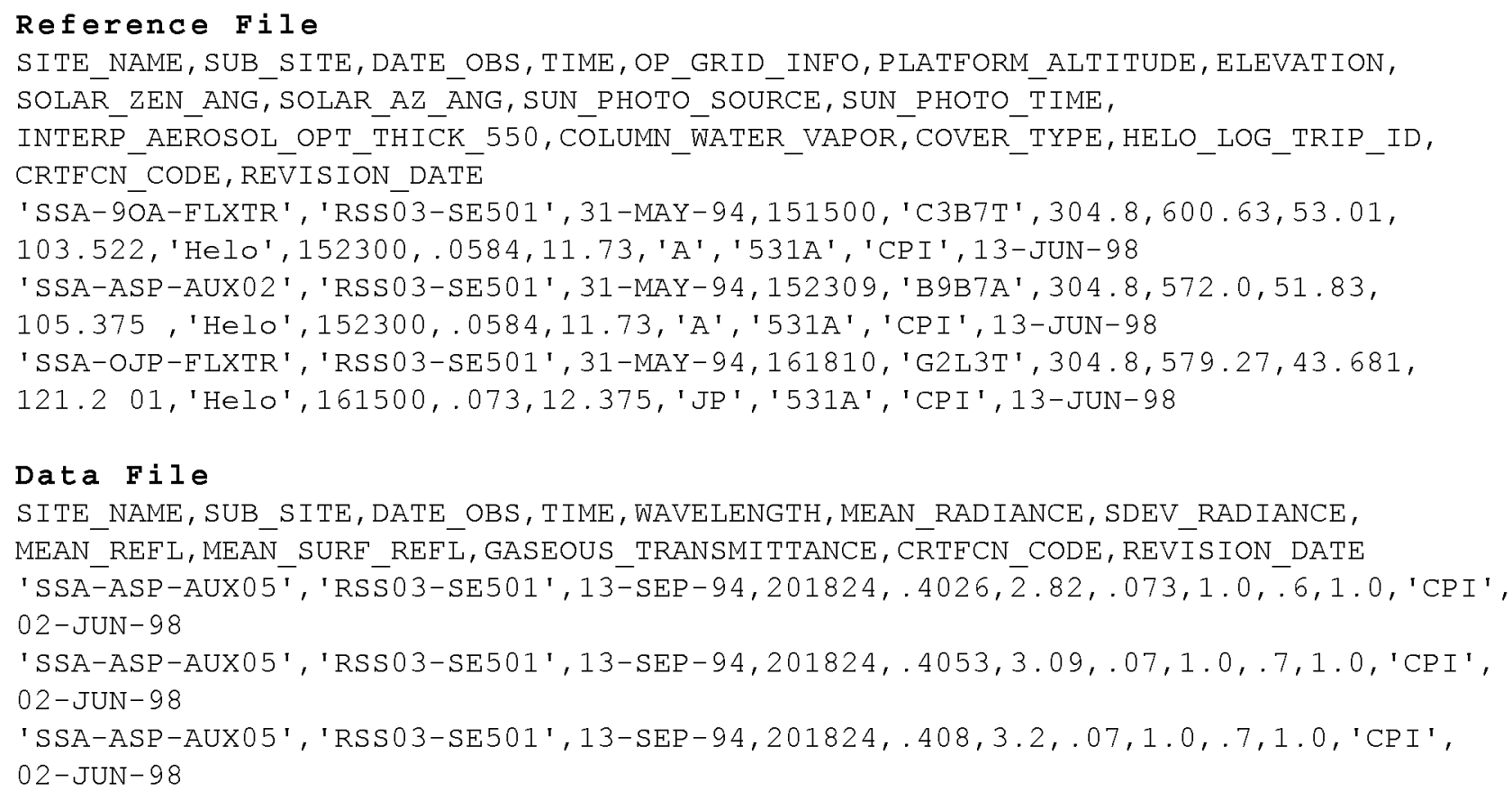

\section{Data Organization}

\subsection{Data Granularity}

The smallest amount of data that can be ordered from this data set is a day's worth of data for a given site.

\subsection{Data Format}

The Compact Disk-Read Only Memory (CD-ROM) files contain American Standard Code for Information Interchange (ASCII) numerical and character fields of varying length separated by commas. The character fields are enclosed with single apostrophe marks. There are no spaces between the fields.

Each data file on the CD-ROM has four header lines of Hyper-Text Markup Language (HTML) code at the top. When viewed with a Web browser, this code displays header information (data set title, location, date, acknowledgments, etc.) and a series of HTML links to associated data files and related data sets. Line 5 of each data file is a list of the column names, and line 6 and following lines contain the actual data. 


\section{Data Manipulations}

\subsection{Formulae}

\subsubsection{Derivation Techniques and Algorithms}

From Vermote et al. (1997):

"Two atmospheric processes modify the solar radiance reflected by a target when viewed from space: absorption by the gases (when observation bands are overlapping gaseous absorption bands) and scattering by the aerosols and the molecules. If the gaseous absorption can be de-coupled from scattering as if the absorbents were located above the scattering layers, as assumed in the $6 \mathrm{~S}$ code, the equation of transfer for a Lambertian homogeneous target of reflectance P_SFC at sea level altitude viewed by a satellite sensor (under zenith angle of view theta_v and azimuth angle of view phi_v) and illuminated by sun (theta_s, phi_s) is...:

$$
\begin{aligned}
& \text { P_TOA(theta_s, theta_v, phi_s-phi_v })=T \text { T } g\left(\text { theta_s, theta_v) } * \left[P \_R+A+T \text { dn }(\text { theta_s }) *\right.\right. \\
& \text { T_up(theta_v) } \left.*\left\{\mathrm{P} \_ \text {SFC } /\left(1-\mathrm{S} * \mathrm{P} \_\mathrm{SFC}\right)\right\}\right] \text {. }
\end{aligned}
$$

The various quantities are expressed in terms of equivalent reflectance $\mathrm{P}$ defined as $\mathrm{P}=\mathrm{pi} * \mathrm{~L} / \mathrm{mu} \_\mathrm{s} *$ E_s where L is the measured radiance, E_s is the solar flux at the top of the atmosphere, and mu_s = cos(theta_s) where theta_s is the solar zenith angle."

In addition, note the following notation (Vermote et al, 1997):

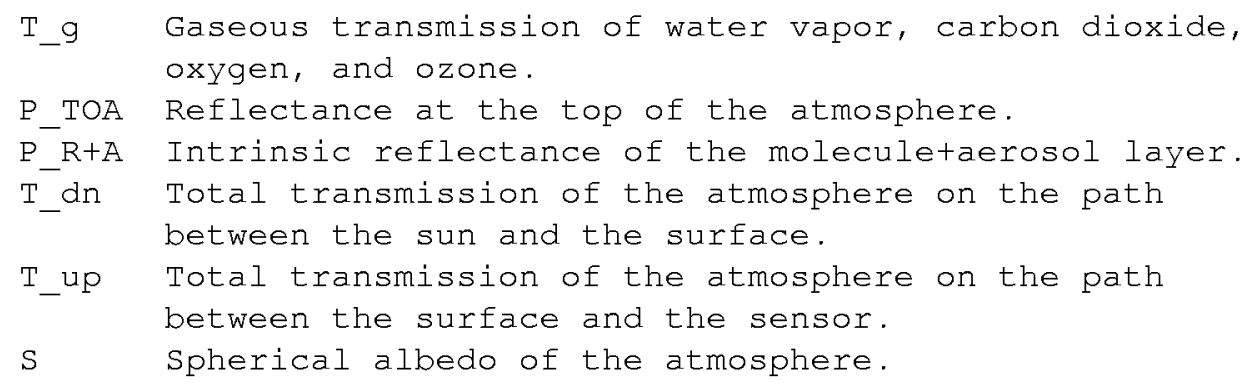

\subsection{Data Processing Sequence}

\subsubsection{Processing Steps}

The SE-590 sensor voltages were processed to at-sensor radiances (W/m² $\mathrm{sr} \mathrm{mm}$ ) following procedures described in Markham et al. (1988). Calibration coefficients were obtained before and after the deployment at NASA GSFC and onsite during the deployment using a portable calibration apparatus. The individual data scans were examined and those with obvious spurious values (i.e., outliers in the distribution) were removed.

The mean helicopter SE-590 radiances and sunphotometer data from either the helicopter or the nearest RSS-11 ground sunphotometer were then input into Version 4.0 of the $6 \mathrm{~S}$ software (Vermote et al., 1997) to obtain at-surface reflectance factors corrected for atmospheric effects. There was close agreement between the helicopter-mounted sunphotometer and nearby RSS-11 measurements.

\subsubsection{Processing Changes}

None.

\subsection{Calculations}

\subsubsection{Special Corrections/Adjustments}

None. 


\subsubsection{Calculated Variables}

See Section 9.1.1.

\subsection{Graphs and Plots}

Not included here. See Loechel et al., 1996.

\section{Errors}

\subsection{Sources of Error}

Potential sources of error include radiometric calibration; spectral calibration; physical (environmental and human) conditions (including helicopter vibration, minor changes in helicopter altitude and inclination); atmospheric conditions, including atmospheric parameters estimated from the surface sunphotometer network; and the atmospheric correction algorithm (Vermote et al., 1997). Confidence intervals for the visible/near-infrared at-sensor radiance values presented in this data set are within $3 \%$. The possibility of errors being introduced into the data set increases with additional manipulations of the data. For an in-depth discussion of error considerations, see Markham et al. (1988).

\subsection{Quality Assessment}

Visual quality assessment was performed during data collection. See reference list and helicopter logs.

\subsubsection{Data Validation by Source}

None given.

\subsubsection{Confidence Level/Accuracy Judgment}

A thorough quantitative error analysis of this kind of data set is given in Markham et al. (1988).

\subsubsection{Measurement Error for Parameters}

Confidence intervals for the at-sensor radiance values presented in this data set are within $3 \%$.

\subsubsection{Additional Quality Assessments}

See helicopter logs. Also, see reference: Walthall et al., 1997.

\subsubsection{Data Verification by Data Center}

A visual examination of all of the helicopter-mounted SE-590 at-surface reflectances reveals artifacts from the atmosphere in all of the spectra. This is especially obvious in the oxygen and water absorption regions in the near-infrared. That all the atmospheric effects have not been removed from the near-infrared bands causes one to suspect the atmospheric corrections in the visible bands as well.

While the atmospheric effects on at-surface reflectance at any given wavelength may be small, these effects may cause significant problems in some types of hyperspectral analyses. Some analysis techniques look at band-to-band covariances or derivatives. The atmospheric effects are nonlinear with wavelength, and thus, by not completely removing these effects, the data become questionable for those types of analyses. 


\section{Notes}

\subsection{Limitations of the Data}

See Section 10.2.5.

\subsection{Known Problems with the Data}

Caution should be used when using a band reflectance in the near-infrared calculated with a gaseous transmittance much under 0.9; i.e., use caution in the oxygen band $(\sim 760 \mathrm{~nm})$ and the water vapor absorption band $(\sim 820 \mathrm{~nm})$-- where absorption effects can be under/overestimated, respectively. It is suggested that a neighboring band not affected by gaseous absorption be used, which seems to characterize surface absorption effects well through the near-infrared.

Data collected over sparse canopies and with extreme solar geometry (i.e., early morning/late afternoon observations) will contain substantial amounts of shadow, which may complicate the retrieval of surface vegetation parameters.

In addition, isolated atmospheric events (such as forest fires or scattered cloudiness) reduce the certainty in the atmospheric correction. The use of surface-measured atmospheric variables contributes to error in the data set in those cases.

\subsection{Usage Guidance}

See Sections 10.2.5, 11.1, and 11.2.

\subsection{Other Relevant Information}

None given.

\section{Application of the Data Set}

Research questions that may be examined with this data include:

- $\quad$ Retrieval of leaf area index (LAI) from spectral vegetation index.

- Scaling of spectral response in boreal regions (in combination with other BOREAS data sets).

\section{Future Modifications and Plans}

None.

\section{Software}

\subsection{Software Description}

The software used in the atmospheric correction of this data set was $6 \mathrm{~S}$, Version 3.2 (Vermote et al., 1997).

\subsection{Software Access}

This software is public domain and available via anonymous ftp at kratmos.gsfc.nasa.gov. 


\section{Data Access}

The RSS-03 reflectance data are available from the Earth Observing System Data and Information System (EOSDIS) Oak Ridge National Laboratory (ORNL) Distributed Active Archive Center (DAAC).

\subsection{Contact Information}

For BOREAS data and documentation please contact:

ORNL DAAC User Services

Oak Ridge National Laboratory

P.O. Box 2008 MS-6407

Oak Ridge, TN 37831-6407

Phone: (423) 241-3952

Fax: (423) 574-4665

E-mail: ornldaac@ornl.gov or orn1@eos.nasa.gov

\subsection{Data Center Identification}

Earth Observing System Data and Information System (EOSDIS) Oak Ridge National Laboratory (ORNL) Distributed Active Archive Center (DAAC) for Biogeochemical Dynamics http://www-eosdis.ornl.gov/ [Internet Link].

\subsection{Procedures for Obtaining Data}

Users may obtain data directly through the ORNL DAAC online search and order system [http://www-eosdis.ornl.gov/] and the anonymous FTP site [ftp://www-eosdis.ornl.gov/data/] or by contacting User Services by electronic mail, telephone, fax, letter, or personal visit using the contact information in Section 15.1.

\subsection{Data Center Status/Plans}

The ORNL DAAC is the primary source for BOREAS field measurement, image, GIS, and hardcopy data products. The BOREAS CD-ROM and data referenced or listed in inventories on the CD-ROM are available from the ORNL DAAC.

\section{Output Products and Availability}

\subsection{Tape Products}

None.

\subsection{Film Products}

None.

\subsection{Other Products}

These data are available on the BOREAS CD-ROM series. 


\section{References}

\subsection{Platform/Sensor/Instrument/Data Processing Documentation}

Markham, B.L., D.L. Williams, J.R. Schafer, F. Wood, and M.S. Kim. 1995. Radiometric

characterization of diode-array field spectroradiometers. Remote Sensing of Environment, vol. 51, pp. 317-330.

\subsection{Journal Articles and Study Reports}

Loechel, S., C.L. Walthall, E. Brown de Colstoun, J. Chen, and B. Markham. 1996. Spatial and temporal variability of surface cover at BOREAS using reflectance from a helicopter platform. International Geosciences and Remote Sensing Symposium (IGARSS), Lincoln, NE.

Loechel, S.E., C.L Walthall, E. Brown de Colstoun, J. Chen, B.L. Markham, and J. Miller. 1997. Variability of boreal forest reflectances as measured from a helicopter platform. Journal of Geophysical Research 102(D24): 29,495-29,503.

Markham, B.L., F.M. Wood Jr., and S.P. Ahmad. 1988. Radiometric calibration of the reflective bands of NS001-thematic mapper simulator (TMS) and modular multispectral radiometers (MMR). In Recent Advances in Sensors Radiometry and Data Processing for Remote Sensing Proc. SPIE 24, pp. 96-108.

Newcomer, J., D. Landis, S. Conrad, S. Curd, K. Huemmrich, D. Knapp, A. Morrell, J. Nickeson, A. Papagno, D. Rinker, R. Strub, T. Twine, F. Hall, and P. Sellers, eds. 2000. Collected Data of The Boreal Ecosystem-Atmosphere Study. NASA. CD-ROM.

Sellers, P. and F. Hall. 1994. Boreal Ecosystem-Atmosphere Study: Experiment Plan. Version 1994-3.0, NASA BOREAS Report (EXPLAN 94).

Sellers, P. and F. Hall. 1996. Boreal Ecosystem-Atmosphere Study: Experiment Plan. Version 1996-2.0, NASA BOREAS Report (EXPLAN 96).

Sellers, P., F. Hall, and K.F. Huemmrich. 1996. Boreal Ecosystem-Atmosphere Study: 1994 Operations. NASA BOREAS Report (OPS DOC 94).

Sellers, P., F. Hall, and K.F. Huemmrich. 1997. Boreal Ecosystem-Atmosphere Study: 1996 Operations. NASA BOREAS Report (OPS DOC 96).

Sellers, P., F. Hall, H. Margolis, B. Kelly, D. Baldocchi, G. den Hartog, J. Cihlar, M.G. Ryan, B. Goodison, P. Crill, K.J. Ranson, D. Lettenmaier, and D.E. Wickland. 1995. The boreal ecosystem-atmosphere study (BOREAS): an overview and early results from the 1994 field year. Bulletin of the American Meteorological Society. 76(9):1549-1577.

Sellers, P.J., F.G. Hall, R.D. Kelly, A. Black, D. Baldocchi, J. Berry, M. Ryan, K.J. Ranson, P.M. Crill, D.P. Lettenmaier, H. Margolis, J. Cihlar, J. Newcomer, D. Fitzjarrald, P.G. Jarvis, S.T. Gower, D. Halliwell, D. Williams, B. Goodison, D.E. Wickland, and F.E. Guertin. 1997. BOREAS in 1997: Experiment Overview, Scientific Results and Future Directions. Journal of Geophysical Research 102(D24): 28,731-28,770.

Strebel, D.E., D.R. Landis, K.F. Huemmrich, and W.W. Meeson. 1994. Collected Data of The First ISLSCP Field Experiment, Volume 1: Surface Observations and Non-Image Data Sets. Published on CD-ROM by NASA.

Vermote, E., D. Tanre, and J. Morcrette. 1997. Second simulation of the satellite signal in the solar spectrum, 6S: an overview. IEEE Trans. Geosci. Remote Sens., vol. 35, no. 3, pp. 675. 
Vermote, E., D. Tanre, J.L. Deuze, M. Herman, and J.J. Morcrette. 1996. Second simulation of the satellite signal in the solar spectrum (6S), 6S User Guide Version 1, October 7, 1996. University of Maryland/Laboratoire d'Optique Atmospherique, 216 pp. (available via anonymous ftp at kratmos.gsfc.nasa.gov).

Walter-Shea, E.A. and L.L. Biehl. 1990. Measuring vegetation spectral properties. Remote Sensing Reviews Chapter 11, Edited by Narendra Goel and John Norman, Vol. 5, pp. 179-205.

Walthall, C. and E. Middleton. 1992. Assessing spatial and seasonal variations in grasslands with spectral reflectances from a helicopter platform. J. Geophys. Res., 97(D17): 18,905-18,912.

Walthall, C., D.L. Williams, B. Markham, J. Kalshoven, and R. Nelson. 1996. Development and present configuration of the NASA GSFC/WFF helicopter-based remote sensing system. International Geosciences and Remote Sensing Symposium (IGARSS). Lincoln, NE.

Walthall, C., S.E. Loechel, K.F. Huemmrich, E. Brown de Colstoun, J. Chen, B. L. Markham, J. Miller, and E.A. Walter-Shea. 1997. Spectral Information Content of the Boreal Forest, 10th International Colloquium on Physical Measurements and Signatures in Remote Sensing, International Society for Photogrammetry and Remote Sensing, Courchevel, France.

\subsection{Archive/DBMS Usage Documentation}

None.

\section{Glossary of Terms}

None.

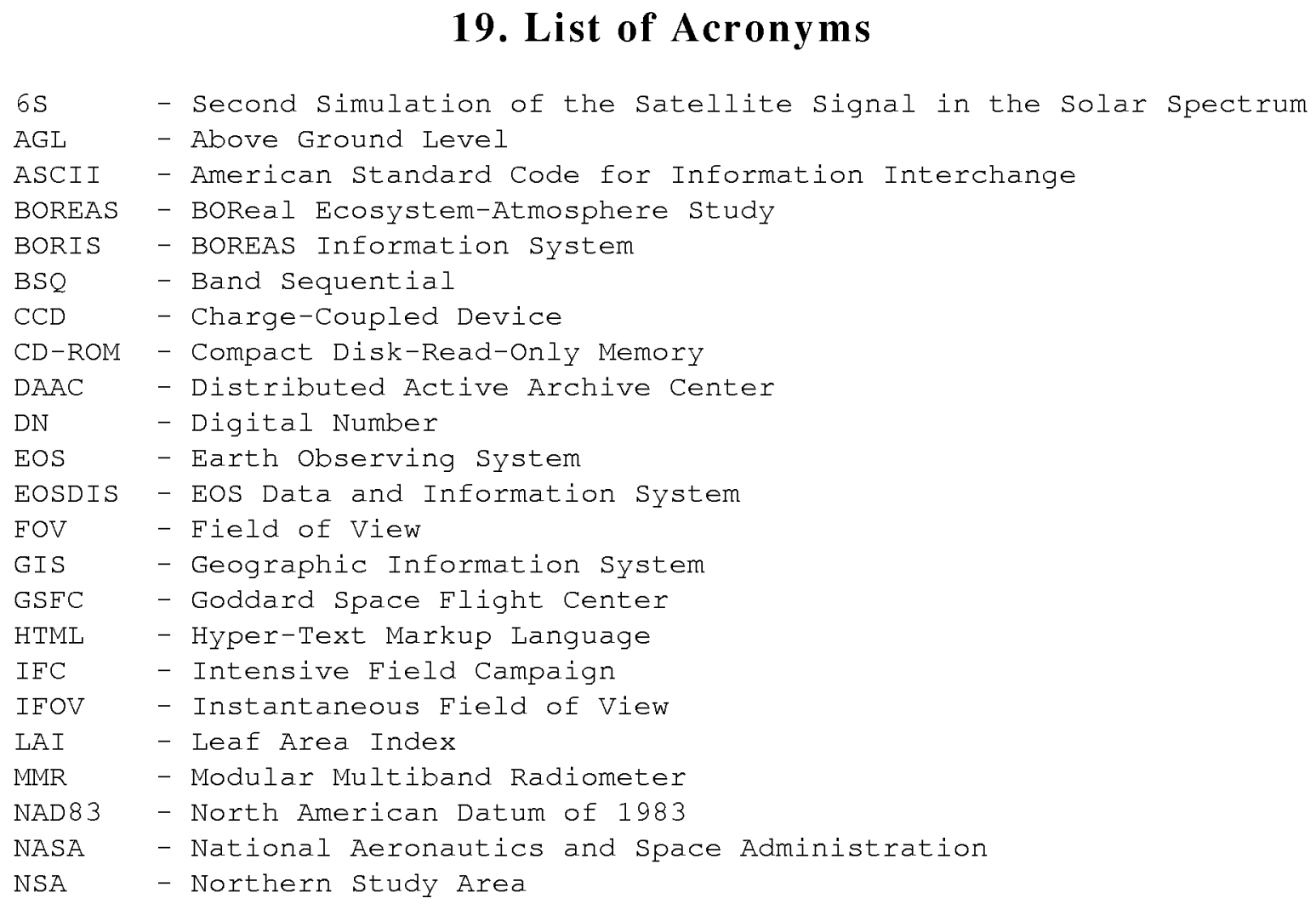




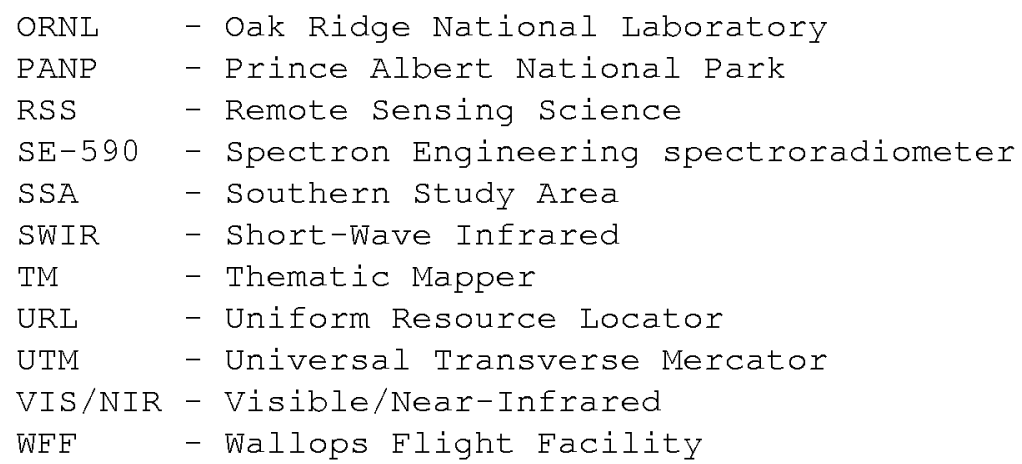

\section{Document Information}

\subsection{Document Revision Date}

Written: 31-Oct-1995

Last Updated: 30-Jul-1999

\subsection{Document Review Date(s)}

BORIS Review: 30-Nov-1997

Science Review: 30-Sep-1998

\subsection{Document ID}

\subsection{Citation}

If this data set is referenced by another investigator, please acknowledge the RSS-03 investigation team and this document. When using these data, please include the following acknowledgement as well as citations of relevant papers in Section 17.2:

The efforts of Dr. Charles Walthall (USDA BARC) and Sara Loechel (University of Maryland) in collecting these data and making them available as well as the efforts of BORIS staff in processing the data are greatly appreciated.

If using data from the BOREAS CD-ROM series, also reference the data as:

Walthall, C. and S. Loechel, "Biophysical Significance of Spectral Vegetation Indices in the Boreal Forest." in Collected Data of The Boreal Ecosystem-Atmosphere Study. Eds. J. Newcomer, D. Landis, S. Conrad, S. Curd, K. Huemmrich, D. Knapp, A. Morrell, J. Nickeson, A. Papagno, D. Rinker, R. Strub, T. Twine, F. Hall, and P. Sellers. CD-ROM. NASA, 2000.

Also, cite the BOREAS CD-ROM set as:

Newcomer, J., D. Landis, S. Conrad, S. Curd, K. Huemmrich, D. Knapp, A. Morrell, J. Nickeson, A. Papagno, D. Rinker, R. Strub, T. Twine, F. Hall, and P. Sellers, eds. Collected Data of The Boreal Ecosystem-Atmosphere Study. NASA. CD-ROM. NASA, 2000.

\subsection{Document Curator}

\subsection{Document URL}


Public reporting burden for this collection of information is estimated to average 1 hour per response, including the time for reviewing instructions, searching existing data sources, gathering and maintaining the data needed, and completing and reviewing the collection of information. Send comments regarding this burden estimate or any other aspect of this collection of information including suggestions for reducing this burden, to Washington Headquarters Services, Directorate for Information Operations and Reports, 1215 Jefferson Davis Highway, Suite 1204, Arlington, VA 22202-4302, and to the Office of Management and Budget, Paperwork Reduction Project (0704-0188), Washington, DC 20503.

\begin{tabular}{l|l|l} 
1. AGENCY USE ONLY (Leave blank) & 2. REPORT DATE & 3. REPORT TYPE AND DATES COVERED
\end{tabular}

\begin{tabular}{l|l|l} 
July 2000 & Technical Memorandum
\end{tabular}

4. TITLE AND SUBTITLE

Technical Report Series on the Boreal Ecosystem-Atmosphere Study (BOREAS)

BOREAS RSS-3 Reflectance Measured from a Helicopter-Mounted SE-590

5. FUNDING NUMBERS

\section{AUTHOR(S)}

Charles L. Walthall and Sara Loechel

Forrest G. Hall and Jaime Nickeson, Editors

7. PERFORMING ORGANIZATION NAME(S) AND ADDRESS (ES)

Goddard Space Flight Center

Greenbelt, Maryland 20771

923

RTOP: $923-462-33-01$
9. SPONSORING / MONITORING AGENCY NAME(S) AND ADDRESS (ES)

National Aeronautics and Space Administration

Washington, DC 20546-0001 8. PEFORMING ORGANIZATION
REPORT NUMBER

2000-03136-0

\section{SUPPLEMENTARY NOTES}

C.L. Walthall: U.S.D.A. Agricultural Research Service; S. Loechel: University of Maryland; J. Nickeson: Raytheon ITSS

\begin{tabular}{l|l}
\hline 12a. DISTRIBUTION / AVAILABILITY STATEMENT & 12b. DISTRIBUTION CODE \\
Unclassified-Unlimited & \\
Subject Category: 43 & \\
Report available from the NASA Center for AeroSpace Information, \\
7121 Standard Drive, Hanover, MD 21076-1320. (301) 621-0390.
\end{tabular}

13. ABSTRACT (Maximum 200 words)

The BOREAS RSS-3 team collected multiple remotely sensed data sets from the NASA UH-1 helicopter. This data set includes helicopter-based radiometric measurements of forested sites acquired during BOREAS made with an SE-590 processed to reflectance factors. The data used in this analysis were collected in 1994 during the three BOREAS IFCs at numerous tower and auxiliary sites in both the NSA and the SSA. The 15-degree FOV of the SE-590 yielded a ground resolution of approximately $79 \mathrm{~m}$ at the $300-\mathrm{m}$ nominal altitude. The data are provided in tabular ASCII files.

\begin{tabular}{l} 
14. SUBJECT TERMS \\
BOREAS, remote sensing science, SE-590. \\
$\begin{array}{l}\text { 17. SECURITY CLASSIFICATION } \\
\text { OF REPORT } \\
\text { Unclassified }\end{array}$ \\
$\begin{array}{c}\text { 18. SECURITY CLASSIFICATION } \\
\text { OF THIS PAGE } \\
\text { Unclassified }\end{array}$ \\
\hline
\end{tabular}

19. SECURITY CLASSIFICATION OF ABSTRACT

Unclassified
15. NUMBER OF PAGES

26

16. PRICE CODE
20. LIMITATION OF ABSTRACT

UL 
